\title{
PAPER
}

\section{The effects of disorder on the normal state and superconducting properties of $\mathrm{Nb}_{3} \mathrm{Sn}$}

To cite this article: M G T Mentink et al 2017 Supercond. Sci. Technol. 30025006

\section{Manuscript version: Accepted Manuscript}

Accepted Manuscript is "the version of the article accepted for publication including all changes made as a result of the peer review process, and which may also include the addition to the article by IOP Publishing of a header, an article ID, a cover sheet and/or an 'Accepted Manuscript' watermark, but excluding any other editing, typesetting or other changes made by IOP Publishing and/or its licensors"

This Accepted Manuscript is @ @ 2016 IOP Publishing Ltd.

During the embargo period (the 12 month period from the publication of the Version of Record of this article), the Accepted Manuscript is fully protected by copyright and cannot be reused or reposted elsewhere.

As the Version of Record of this article is going to be / has been published on a subscription basis, this Accepted Manuscript is available for reuse under a CC BY-NC-ND 3.0 licence after the 12 month embargo period.

After the embargo period, everyone is permitted to use copy and redistribute this article for non-commercial purposes only, provided that they adhere to all the terms of the licence https://creativecommons.org/licences/by-nc-nd/3.0

Although reasonable endeavours have been taken to obtain all necessary permissions from third parties to include their copyrighted content within this article, their full citation and copyright line may not be present in this Accepted Manuscript version. Before using any content from this article, please refer to the Version of Record on IOPscience once published for full citation and copyright details, as permissions will likely be required. All third party content is fully copyright protected, unless specifically stated otherwise in the figure caption in the Version of Record.

View the article online for updates and enhancements. 


\title{
The Effects of Disorder on the Normal State and Superconducting Properties of $\mathrm{Nb}_{3} \mathrm{Sn}$
}

\author{
M. G. T. Mentink ${ }^{1}$, M. M. J. Dhalle ${ }^{2}$, D. R. Dietderich ${ }^{3}$, \\ A. Godeke ${ }^{4}$, F. Hellman ${ }^{5}$, and H. H. J. ten Kate ${ }^{1,2}$ \\ ${ }^{1}$ CERN, CH-1211 Geneva 23, Switzerland \\ ${ }^{2}$ University of Twente, Enschede 7500 AE, The Netherlands \\ ${ }^{3}$ Lawrence Berkeley National Laboratory, 1 Cyclotron Rd, MS 47R0112, Berkeley, \\ CA 94720, USA \\ ${ }^{4}$ National High Field Laboratory, 1800 E. Paul Dirac Dr., Tallahassee, FL32310-3706, \\ USA \\ ${ }^{5}$ University of California, Berkeley, 366 LeConte Hall MC 7300, Berkeley, CA, \\ 94720-7300, USA
}

E-mail: mmentink@cern.ch

\begin{abstract}
The effect of disorder on the normal state resistivity and the superconducting properties of $\mathrm{Nb}_{3} \mathrm{Sn}$ is explored in a combination of ab-initio calculations and microscopic theory.

The crystal symmetry is calculated to be preferentially tetragonal at a normal state resistivity below $27.0 \pm 1.4 \mu \Omega \mathrm{cm}$, and preferentially cubic above this value, which is shown to be consistent with the experimentally observed transition point. The phonon density of states, the Eliashberg spectrum $\alpha^{2}(\omega) F(\omega)$, the electron-phonon coupling constant, the characteristic frequency, the critical temperature $T_{\mathrm{c}}$, and the upper critical magnetic field at $0 \mathrm{~K} \mathrm{H}_{\mathrm{c} 2}(0)$ are calculated over a large normal state resistivity range and shown to be consistent with experimental observations.

The high degree of consistency between the calculation results and experimental observations is a strong indication that the calculation approach utilized here, a combination of ab-initio calculations and microscopic theory, is useful tool for understanding the superconducting and normal state properties of $\mathrm{Nb}_{3} \mathrm{Sn}$.
\end{abstract}

\section{Introduction}

Since the discovery of superconductivity in $\mathrm{Nb}_{3} \mathrm{Sn}$ by Matthias et al. [1] in 1954, this material has attracted a significant amount of scientific attention, not just because of the exotic properties of the material, but also for the suitability of the material for large-scale high-magnetic field applications, such as the High Luminosity Large Hadron Collider, the International Thermonuclear Experimental Reactor, Nuclear Magnetic Resonance magnets, and high-magnetic field facilities. $\mathrm{Nb}_{3} \mathrm{Sn}$ is well suited for these applications because $\mathrm{Nb}_{3} \mathrm{Sn}$ wires can be produced and bundled into cables in a reliable and relatively affordable manner. The superconducting properties of $\mathrm{Nb}_{3} \mathrm{Sn}$ exceed the 
superconducting properties of NbTi, which is another commonly used superconductor for medium-magnetic field applications.

Inspite of previous scientific research spanning several decades, the superconductor $\mathrm{Nb}_{3} \mathrm{Sn}$ still has features that are not yet fully understood, such as the profound degree by which strain affects the superconducting properties of the material. In addition to strain, the degree of disorder also strongly affects the superconducting properties of $\mathrm{Nb}_{3} \mathrm{Sn}$ (see for instance Orlando et al. [2]). In this paper, a number of phenomena of $\mathrm{Nb}_{3} \mathrm{Sn}$ are considered, which include the preferential crystal symmetry, the Eliashberg spectra, the critical temperature, and the upper critical magnetic field as a function of disorder. The behavior is calculated through the use of ab-initio calculations and subsequently validated through previously published experimental observations. The purposes of this work are to provide a thorough review of existing literature on the microscopic properties of $\mathrm{Nb}_{3} \mathrm{Sn}$, to combine microscopic theory with density functional theory calculations, and to validate calculation results for the purpose of demonstrating the applicability of the approach for understanding the exotic properties of $\mathrm{Nb}_{3} \mathrm{Sn}$. For future purposes, the calculation approach taken here could be used to investigate specific phenomena of superconducting $\mathrm{Nb}_{3} \mathrm{Sn}$, such as the large intrinsic strain sensitivity of its superconducting properties (also see [3]).

Firstly, three starting assumptions are discussed in section 2. Secondly, a variety of calculation results pertaining to normal state properties of $\mathrm{Nb}_{3} \mathrm{Sn}$ are compared to experimental observations in section 3 for the purpose of providing empirical validation for assumptions given in section 2. This comparison includes results regarding the martensitic transformation, the electron density of states, the Fermi velocity, the phonon density of states, the Eliashberg spectrum, the electron-phonon coupling constant, and the characteristic phonon frequency. Thirdly, calculation results are shown side-by-side with experimental observations on the superconducting phase boundary in section 4 , which include the critical temperature, the upper critical field and a discussion on the influence of the martensitic transformation on the critical temperature and upper critical field. Finally, this is followed by a discussion in section 5 and conclusions in section 6 .

\section{Starting assumptions}

\subsection{Assumption \#1: Electron lifetime broadening approximation}

In order to study the influence of disorder in the ab-initio calculations, a previously published concept after Testardi and Mattheiss [4, 5] was used. In this concept, disorder is introduced through electron lifetime broadening. The underlying idea is that the energy of an electron in a disordered material is poorly defined in comparison to the energy of an electron in a perfect crystal, because the electron in the disordered material scatters more often. The degree of uncertainty, i.e. broadening, is expressed with [5]:

$$
E_{\mathrm{b}}=\frac{\hbar}{\tau}
$$



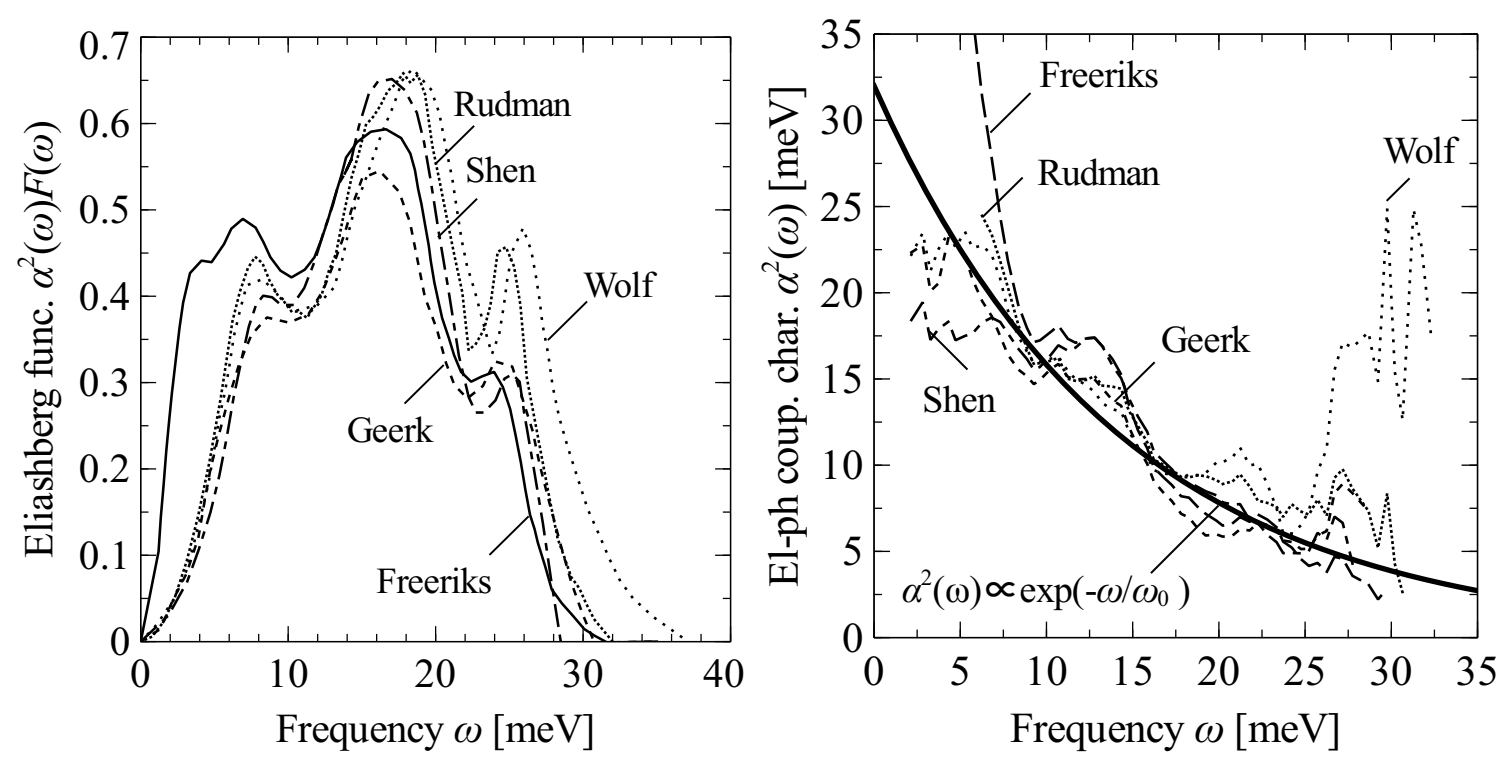

Figure 1. Left: Experimentally observed Eliashberg function $\alpha^{2}(\omega) F(\omega)$ as a function of vibrational frequency $\omega$ of low-resistivity $\mathrm{Nb}_{3} \mathrm{Sn}$, determined from stoichiometric tunnel junctions, after Freeriks et al. [11], Rudman et al. [8], Shen [9], Geerk et al. [10], and Wolf [12]. Right: Experimentally observed Eliashberg function divided by phonon density of states, after Schweiss et al. [13], and empirical exponential fit.

where $E_{\mathrm{b}}$ is the broadening energy in $[\mathrm{eV}], \hbar$ is the reduced Planck constant in $[\mathrm{eV} \times \mathrm{s}]$, and $\tau$ is the mean scattering time in $[\mathrm{s}]$. Thus, disorder is incorporated through a mean scattering time $\tau$, where a smaller $\tau$ indicates a higher degree of disorder.

This broadening term is subsequently introduced in density functional theory and density functional perturbation theory calculations, through which the effect of disorder on a stoichiometric unit cell, i.e. two Sn ions and six Nb ions, is investigated.

The validity of this assumption is empirically demonstrated through a variety of comparisons between calculation results and experimental observations, in particular with regards to the disorder-dependent occurrence of the martensitic transformation in section 3.2 .

\subsection{Assumption \#2: Empirical $\alpha^{2}(\omega)$ dependence}

A second underlying assumption involves the electron-phonon coupling characteristic $\alpha^{2}$. The relevance of $\alpha^{2}(\omega)$ is that it relates the electron-phonon coupling constant $\lambda$ to the electronic and vibrational properties of the material. $\lambda$ is related to the phonon density of states $F(\omega)$ through [6]:

$$
\lambda=2 \int \frac{\alpha^{2}(\omega) F(\omega)}{\omega} \delta \omega,
$$

where $\alpha^{2}(\omega)$ is the electron-phonon coupling characteristic, $\omega$ the vibrational frequency, and $F(\omega)$ the phonon density of states. It was empirically demonstrated by Markiewicz 
[7] that, in stoichiometric $\mathrm{Nb}_{3} \mathrm{Sn}, \alpha^{2}(\omega)$ is proportional to an exponential function:

$$
\alpha^{2}(\omega)=\alpha_{0} \exp \left(-\omega / \omega_{0}\right),
$$

where $\omega_{0}$ is a characteristic frequency and $\alpha_{0}$ is a constant. Analogous to the method as used by Markiewicz, $\alpha^{2}(\omega)$ was determined from published measurements of the Eliashberg spectrum $\alpha^{2}(\omega) F(\omega)$ (after Rudman et al. [8], Shen [9], Geerk et al. [10], Freeriks et al. [11], and Wolf [12]) and the phonon density of states $F(\omega)$ (after Schweiss et al. [13]), as shown in fig. 1. These results are then fitted with equation 3 to find a value of $14.2 \mathrm{meV}$ for $\omega_{0}$ and $32.0 \mathrm{meV}$ for $\alpha_{0}$.

According to McMillan [14] and Hopfield [15] $\lambda$ is proportional to the electron density of states $N\left(E_{\mathrm{F}}\right)$ through:

$$
\lambda=\frac{N\left(E_{\mathrm{F}}\right)\left\langle I^{2}\right\rangle}{\left\langle\omega^{2}\right\rangle M}
$$

where $\left\langle I^{2}\right\rangle$ is the mean electronic matrix element, $M$ the effective ion mass, and $\left\langle\omega^{2}\right\rangle$ the average of the squared vibrational frequency in the Eliashberg spectrum. The phonon density of states is a normalized quantity, i.e.:

$$
\int F(\omega) \delta \omega \equiv 1
$$

The definition of $\left\langle\omega^{2}\right\rangle$ is as follows:

$$
\left\langle\omega^{2}\right\rangle \equiv \frac{2}{\lambda} \int \alpha^{2}(\omega) F(\omega) \omega \times \delta \omega
$$

When combining equations $2,4,5$, and 6 , a solution for $\alpha^{2}(\omega)$ is found for a material with a single ion species:

$$
\alpha^{2}(\omega)=\frac{\left\langle I^{2}\right\rangle}{2 M} \frac{N\left(E_{\mathrm{F}}\right)}{\omega}=\alpha_{\mathrm{IM}}^{2} \frac{N\left(E_{\mathrm{F}}\right)}{\omega},
$$

where $\alpha_{\mathrm{IM}}^{2}$ is a constant. However, $\mathrm{Nb}_{3} \mathrm{Sn}$ contains two different ion species. As argued by Schweiss [13], $\lambda$ can be approximated as the sum of the contributions of the various ions, through:

$$
\lambda_{\text {eff }}=\lambda_{1}+\lambda_{2}+\ldots
$$

The total phonon density of states is expressed as a sum of the projected phonon densities of states, i.e. the sum of contributions from individual ions:

$$
F(\omega)=F_{1}(\omega)+F_{2}(\omega)+\ldots
$$

The effective electron-phonon coupling characteristic may then be expressed in terms of the various constituents, with:

$$
\begin{aligned}
& \alpha_{\mathrm{eff}}^{2}(\omega) F(\omega)=\frac{N\left(E_{\mathrm{F}}\right)}{\omega}\left(\alpha_{\mathrm{IM} 1}^{2} F_{1}(\omega)+\alpha_{\mathrm{IM} 2}^{2} F_{2}(\omega)+\ldots\right), \\
& \alpha_{\mathrm{eff}}^{2}(\omega)=\frac{N\left(E_{\mathrm{F}}\right)}{\omega} \frac{\alpha_{\mathrm{IM} 1}^{2} F_{1}(\omega)+\alpha_{\mathrm{IM} 2}^{2} F_{2}(\omega)+\ldots}{F(\omega)} .
\end{aligned}
$$


A key point is that while equation 7 implies that $\alpha^{2}$ may be expressed with a simple analytical equation, in the case of multiple ion species $\alpha^{2}$ depends on the relative contributions of the various ions, so that an analytical deduction is no longer trivial. To account for the relative contributions of the various ions, a weighting function is introduced:

$$
\begin{aligned}
& \alpha_{\mathrm{IM}, \mathrm{eff}}^{2} f(\omega)=\frac{\alpha_{\mathrm{IM} 1}^{2} F_{1}(\omega)+\alpha_{\mathrm{IM} 2}^{2} F_{2}(\omega)+\ldots}{F(\omega)}, \\
& \alpha_{\mathrm{eff}}^{2}(\omega)=\frac{N\left(E_{\mathrm{F}}\right)}{\omega} \alpha_{\mathrm{IM}, \mathrm{eff}}^{2} f(\omega) .
\end{aligned}
$$

Here $\alpha_{\text {IM,eff }}^{2}$ is a constant related to the effective ion mass and the mean electronic matrix element and $f(\omega)$ is a weighting function.

The value of $N\left(E_{\mathrm{F}}\right)$ of stoichiometric $\mathrm{Nb}_{3} \mathrm{Sn}$ is taken at 15.4 states / $(\mathrm{eV} \times$ unit cell) according to Schachinger et al. [16], which is consistent with literature results by Orlando et al. [17] and Ghosh et al. [18], also see section 3.3. With the given value of $N\left(E_{\mathrm{F}}\right)$ in stoichiometric $\mathrm{Nb}_{3} \mathrm{Sn}$, the value of $\alpha_{\mathrm{IM} \text {,eff }}^{2}$ and the frequency-dependent $\left.f_{(} \omega\right)$ may be derived from empirical observations. Combining equations 3 and 13, a value of $2.08 \times 10^{-3} \mathrm{eV}^{2} /\left(\right.$ states $\times$ unit cell) is found for $\alpha_{\mathrm{IM}, \mathrm{eff}}^{2}$, and $f(\omega)$ is expressed as:

$$
f(\omega)=\omega \exp \left(-\omega / \omega_{0}\right) .
$$

$f(\omega)$ is a function which peaks at the $\omega_{0}$, which is equal to $14.2 \mathrm{meV}$. This result is consistent with the analysis by Schweiss et al. [13], who pointed out the relatively large contribution of phonons to the intermediate frequency range of the Eliashberg spectrum, and attributed this result to the relatively large degree of electron-phonon coupling of niobium with respect to tin. In other words, Markiewicz's empirical description of the electron-phonon coupling characteristic may be understood in terms of the relative contributions of two different types of ions. Combining equations 13 and 14 we find an empirical expression of $\alpha_{\text {eff }}^{2}(\omega)$ which is:

$$
\alpha_{\text {eff }}^{2}(\omega)=\alpha_{\mathrm{IM}, \mathrm{eff}}^{2} N\left(E_{\mathrm{F}}\right) \exp \left(-\omega / \omega_{0}\right)
$$

where $\alpha_{\mathrm{IM} \text {,eff }}^{2}$ is equal to $2.08 \times 10^{-3} \mathrm{eV}^{2} /$ (states $\times$ unit cell) and $\omega_{0}$ is equal to $14.2 \mathrm{meV}$.

To summarize, an attempt was made to analytically derive the expression of $\alpha_{\text {eff }}^{2}$ but it was found that even when $\alpha^{2}$ is known for a material with a single ion species, finding an analytical expression for a more complex material such a $\mathrm{Nb}_{3} \mathrm{Sn}$ is non-trivial. Therefore, the next best thing is done: To combine microscopic theory with experimental observations on stoichiometric $\mathrm{Nb}_{3} \mathrm{Sn}$ and find an empirical expression of $\alpha_{\text {eff }}^{2}(\omega)$, where $\alpha_{\text {IM,eff }}^{2}$ and $\omega_{0}$ are treated as global constants. The validity of this approach is empirically justified in section 3.4. 


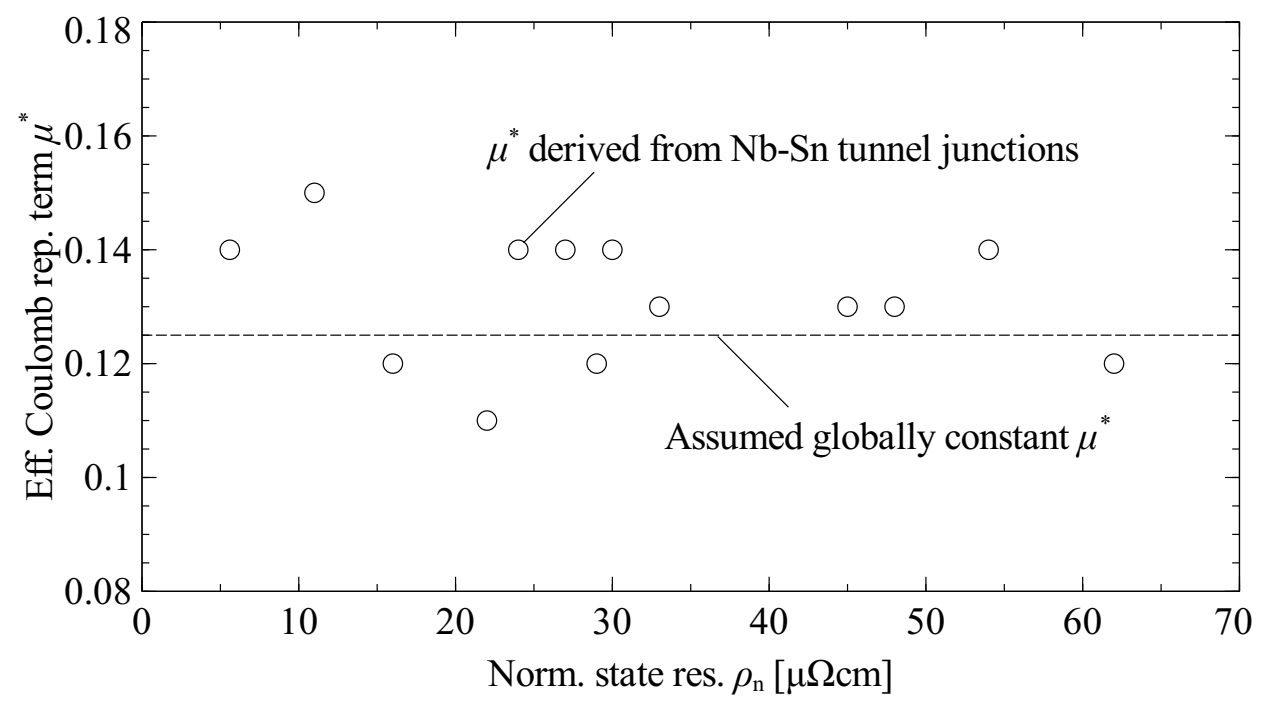

Figure 2. Effective Coulomb repulsion term $\mu^{*}$ as a function of $\rho_{\mathrm{n}}$ of Nb-Sn after Rudman et al. [8] and assumed globally constant $\mu^{*}$.

\subsection{Assumption \#3: Constant effective coulomb repulsion term $\mu^{*}$}

A third starting assumption pertains to the effective coulomb repulsion term. The proper value of $\mu^{*}$ is determined in two separate manners.

Firstly, $\mu^{*}$ in both stoichiometric and off-stoichiometric Nb-Sn was previously experimentally determined by Rudman et al. [8]. From fig. 2 it is clear that $\mu^{*}$ stays within the $0.13 \pm 0.02$ range over the entire normal state resistivity range.

Secondly, the Eliashberg spectra of several stoichiometric low-resistivity $\mathrm{Nb}_{3} \mathrm{Sn}$ samples were previously published and the critical temperature of stoichiometric $\mathrm{Nb}_{3} \mathrm{Sn}$ is known to be about $18 \mathrm{~K}$. The critical temperature is related to the Eliashberg spectrum and $\mu^{*}$. Two commonly used expressions, both of which incorporate strong-coupling corrections, are the critical temperature expressions by Kresin [19] and by Allen and Dynes [20], with:

$$
\begin{aligned}
& T_{\mathrm{c}, \mathrm{Kresin}}=\frac{0.25\left\langle\omega^{2}\right\rangle^{0.5}}{\sqrt{e^{2 / \lambda_{\text {eff }}}-1}}, \\
& T_{\mathrm{c}, \mathrm{AD}}=\frac{f_{1} f_{2} \omega_{\mathrm{log}}}{1.2} \exp \left(\frac{-1.04(1+\lambda)}{\lambda-\mu^{*}-0.62 \lambda \mu^{*}}\right),
\end{aligned}
$$

where $\lambda_{\text {eff }}, f_{1}, f_{2}$, and $\omega_{\log }$ are related to $\lambda, \mu^{*}$, and the Eliashberg spectrum. For brevity, the full sets of equations are not reproduced here, but the reader is referred to the original papers.

With both the Eliashberg spectrum and critical temperature of stoichiometric lowresistivity known, the only unknown parameter is $\mu^{*}$, which may then be calculated for each measurement and each of the two expressions. The result of this is shown in table 1. The average of $\mu^{*}$, deduced for five published Eliasberg spectrum and using both equations is 0.125 , with a standard deviation of 0.04 . This value is taken as a global 
Table 1. Values for $\lambda,\left\langle\omega^{2}>^{0.5}\right.$ and $\mu^{*}$ of weakly disordered stoichiometric $\mathrm{Nb}_{3} \mathrm{Sn}$ after Rudman et al. [8], Shen [9], Geerk et al. [10], Freeriks et al. [11], and Wolf [12], mean values, and standard deviations.

\begin{tabular}{ccccc}
\hline & $\lambda$ & $\left\langle\omega^{2}\right\rangle^{0.5}[\mathrm{meV}]$ & $\mu_{\text {Kresin }}^{*}$ & $\mu_{\text {Allen-Dynes }}^{*}$ \\
\hline Freeriks & 2.55 & 10.9 & 0.17 & 0.17 \\
Wolf & 1.79 & 15.2 & 0.16 & 0.15 \\
Shen & 1.56 & 13.9 & 0.09 & 0.11 \\
Geerk & 1.50 & 13.8 & 0.08 & 0.06 \\
Rudman & 1.75 & 14.2 & 0.13 & 0.12 \\
\hline Average & 1.83 & 13.6 & 0.127 & 0.122 \\
Std.dev & 0.42 & 1.6 & 0.042 & 0.044 \\
\hline
\end{tabular}

constant, which is found to be consistent with the experimental observations over the entire investigated resistivity range (fig. 2).

\section{Comparison of calculated versus experimentally observed normal state properties}

\subsection{Density Functional Theory (DFT) and Density Functional Perturbation Theory (DFPT) calculations}

With these three assumptions, a series of DFT and DFPT calculations are performed using Quantum Espresso [21], an open source software suite for electron-structure calculations. The purpose is to allow for comparisons between calculation results and experimental observations.

DFT and DFPT calculations are performed on a simulated stoichiometric $\mathrm{Nb}_{3} \mathrm{Sn}$ unit cell, consisting of six niobium and two tin atoms, which are arranged in the A15 crystal structure (fig.3, left graph). Quantum Espresso uses a plane waves basis set and pseudopotentials. Perdew-Wang 91 gradient corrected functional approximation pseudopotentials [22] are used, and they are readily available on the Quantum Espresso website [23]. All calculations were performed with a kinetic energy cutoff of 40 Rydberg (540 eV) and a charge density cutoff of 320 Rydberg (4350 eV). Structural optimization and density of states calculations were performed with a k-point grid consisting of $16 \times 16 \times 16$ automatic Monkhorst-Pack divisions. Fermi velocity calculations were performed with a k-point grid of $40 \times 40 \times 40$ automatic Monkhorst-Pack divisions. The phonon calculations were performed with a k-point grid consisting of $8 \times 8 \times 8$ MonkhorstPack divisions and a q-point grid consisting of $4 \times 4 \times 4$ Monkhorst-Pack divisions. Following Testardi [4], Fermi-Dirac broadening is utilized in all calculations, where the 

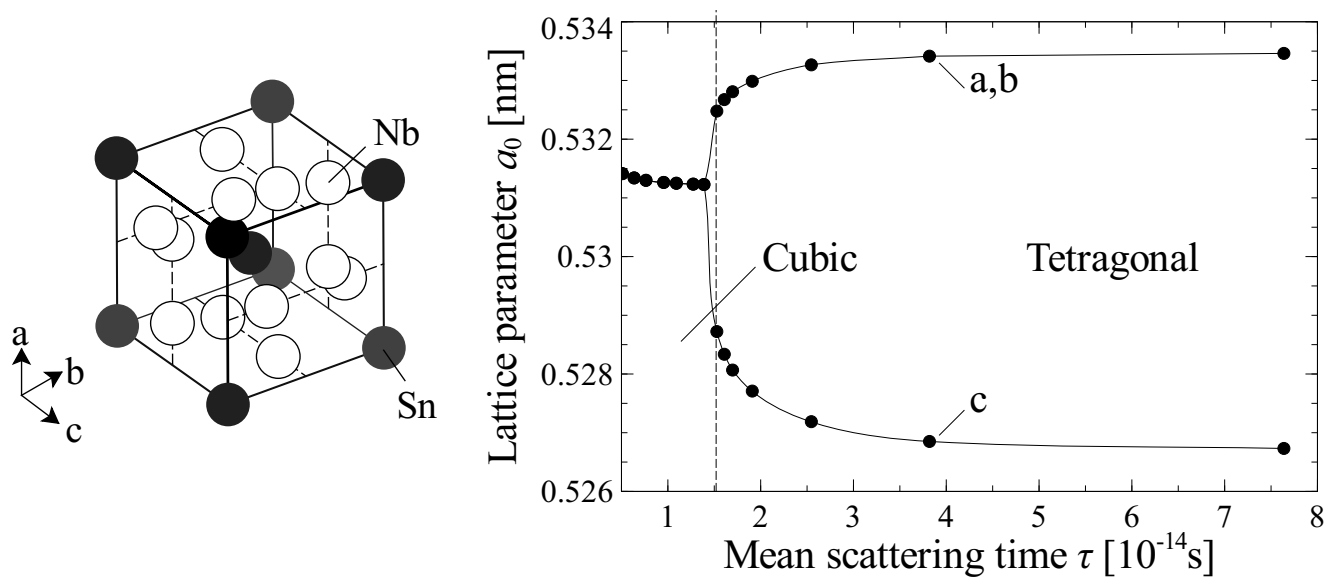

Figure 3. Left: A15 crystal structure of $\mathrm{Nb}_{3} \mathrm{Sn}$. Right: mean scattering time dependent optimal lattice parameters.

amount of broadening is given by equation 1 .

\subsection{Occurrence of the Martensitic transformation}

The martensitic transformation is a phenomenon in which the crystal structure spontaneously transforms from cubic to tetragonal upon cooling below the martensitic transformation temperature $T_{\mathrm{M}}$ in low-resistivity $\mathrm{Nb}_{3} \mathrm{Sn}$. This phenomenon was studied in detail by Mailfert et al. [24], Arko et al. [25], Watanabe et al. [26], Axe et al. [27], and Mentink et al. [28] in which the effect of the martensitic transformation on the normal state resistivity $\rho_{\mathrm{n}}$ and the phonon properties of low-resistivity $\mathrm{Nb}_{3} \mathrm{Sn}$ were investigated. In these analyses, the martensitic transformation temperature $T_{\mathrm{M}}$ was determined at $45 \pm 7 \mathrm{~K}$, which corresponds to a normal state resistivity of $27 \pm 3 \mu \Omega \mathrm{cm}$ $[28]$.

Devantay et al. [29] and Zhou et al. [30] performed studies of binary Nb-Sn bulk samples with a variety of tin concentrations. In both investigations the normal state resistivity at $T_{\mathrm{c}}$, the composition, and the effect of the martensitic transformation on selected X-ray diffraction peaks at room temperature and at $10 \mathrm{~K}$ was determined. According to Devantay et al., a sample with an average tin concentration of 24.4 at.\% and a normal state resistivity of $19 \mu \Omega \mathrm{cm}$ is a mix of cubic and tetragonal $\mathrm{Nb}_{3} \mathrm{Sn}$ at $10 \mathrm{~K}$, while in a sample with a tin concentration of 23.9 at.\% and a normal state resistivity of $23 \mu \Omega \mathrm{cm}$, no indication of tetragonal distortion was observed. In the investigation by Zhou et al., predominantly tetragonal $\mathrm{Nb}_{3} \mathrm{Sn}$ was observed in three samples with normal state resistivities below $20.9 \mu \Omega \mathrm{cm}$, while the material was shown to be almost entirely cubic for samples with normal state resistivities above $31.3 \mu \Omega \mathrm{cm}$ (not including a sample with an unusually high normal state resistivity, which the author attributed to $\mathrm{NbSn}_{2}$ grain boundary precipitation). In experiments by Goldacker et al. [31, 32], it was observed that the martensitic transformation in stoichiometric $\mathrm{Nb}_{3} \mathrm{Sn}$ could be prevented 
through various additions including tantalum, titanium, nickel, gallium, and hydrogen. The martensitic transformation was completely suppressed in samples with 0.6 at.\% $\mathrm{H}$, 2.8 at. $\% \mathrm{Ta}, 1.3$ at.\% Ti, and 1 at.\% Ni, with normal state resistivities of $37 \mu \Omega \mathrm{cm}$, $29 \mu \Omega \mathrm{cm}, 33 \mu \Omega \mathrm{cm}$, and $30 \mu \Omega \mathrm{cm}$, respectively. The martensitic transformation did, however, occur in a binary bulk sample, also see Guritanu et al. [33], and a sample with 0.9 at.\% Ga, with normal states resistivities of 13 and $23 \mu \Omega \mathrm{cm}$. The martensitic transformation temperature range of 20 to $50 \mathrm{~K}$ was observed in a sample with 1.7 at.\% Ta and a normal state resistivity of $26 \mu \Omega \mathrm{cm}$, and it was concluded that 1.7 at.\% is very close to the critical tantalum concentration that is required to suppress the martensitic transformation.

In summary, the literature results show that there is a critical value of resistivity (25 $\pm 3 \mu \Omega \mathrm{cm}$ ), above which the material remains cubic (i.e. the martensitic transformation is suppressed) and below which the transformation occurs, independently of whether this resistivity is caused by off-stoichiometry, the presence of ternary impurities, or a non-zero temperature.

The effect of disorder on the optimal crystal structure is investigated here by performing structural optimizations at various values of $\tau$, where $\tau$ is indicative of the degree of disorder. The positions of the ions inside the crystal as well as the lattice parameters are optimized simultaneously, under the assumption of no externally applied stress. The structural optimizations start from an orthorhombic configuration and are found to converge to either a cubic or a tetragonal crystal symmetry (see fig. 3, right graph). The calculation results indicate that the crystal is preferentially cubic at $\tau<\tau_{\mathrm{c}}$ and tetragonal at $\tau>\tau_{\mathrm{c}}$, and the transition between cubic and tetragonal crystal symmetry is found to occur at a critical mean scattering time $\tau_{\mathrm{c}}$ of $(1.53 \pm 0.08) \times 10^{-14} \mathrm{~s}$.

Whenever the optimal crystal structure at a given $\tau$ is tetragonal, the calculation result additionally indicates sublattice distortion in the niobium chains, a phenomenon that was previously studied from a theoretical perspective by Labbé et al. [34], from a computational perspective by Sadigh et al. [35] and Weber et al. [36], and from an experimental perspective by Shirane et al. [37]. Conversely, no sublattice distortion is observed whenever the optimal crystal structure is cubic.

Following Testardi et al. [4], Mattheis et al. [5, 38], Schachinger et al. [16], and Allen et al. [39, 40, 41], the normal state resistivity is calculated through an expression which relates $\rho_{\mathrm{n}}$ to $N\left(E_{\mathrm{F}}\right), v_{\mathrm{F}}, \tau$, and $V$, with:

$$
\rho_{\mathrm{n}, \mathrm{x}}=\frac{V}{e N\left(E_{\mathrm{F}}\right) v_{\mathrm{F}, \mathrm{x}}^{2} \tau},
$$

where $\rho_{\mathrm{n}, \mathrm{x}}$ is the normal state resistivity in direction $x$ in $[\Omega \mathrm{m}], V$ the molecular volume $\left[\mathrm{m}^{3}\right], e$ is elementary charge in $[\mathrm{C}], N\left(E_{\mathrm{F}}\right)$ is electron density of states at the Fermi energy in $\left[\right.$ states $\times \mathrm{eV}^{-1} \times$ unit cell ${ }^{-1}$, and $v_{\mathrm{F}, \mathrm{x}}$ the root mean square Fermi velocity in direction $x$ in $\left[\mathrm{ms}^{-1}\right]$. According to Schachinger [16], this expression is valid for $\mathrm{Nb}_{3} \mathrm{Sn}$ under the assumption that the normal state resistivity is dominated by s-wave scattering of non-magnetic impurities. Following Pickett et al. [42], the root mean square Fermi 
velocity is calculated:

$$
\begin{aligned}
& V=a_{\mathrm{x}} a_{\mathrm{y}} a_{\mathrm{z}}, \\
& \left(\hbar v_{\mathrm{F}, \mathrm{x}}\right)^{2}=\frac{\sum_{k, n}\left(E_{\mathrm{x}}(k, n)^{2} \delta\left(E(k, n)-E_{\mathrm{F}}\right)\right)}{\sum_{k, n} \delta\left(E(k, n)-E_{\mathrm{F}}\right)}, \\
& E_{\mathrm{x}}(k, n)=\frac{E\left(k+\Delta k_{\mathrm{x}}, n\right)-E(k, n)}{\Delta k_{\mathrm{x}}}, \\
& \delta(E)=\frac{1}{2+\exp \left(\frac{E}{\hbar \tau}\right)+\exp \left(\frac{-E}{\hbar \tau}\right)},
\end{aligned}
$$

where $k$ is a three-dimensional coordinate in momentum-space in $\left[\mathrm{m}^{-1}, \mathrm{~m}^{-1}, \mathrm{~m}^{-1}\right], n$ the band number, $\Delta k$ the distance between two $k$-space coordinates in $\left[\mathrm{m}^{-1}\right], E$ the energy of electron band $n$ at momentum-space coordinate $k$ in $[\mathrm{eV}], x$ a specific direction in momentum space, $\hbar$ the reduced Planck constant in $[\mathrm{eV} \times \mathrm{s}]$, and $v_{\mathrm{F}, \mathrm{x}}$ the Fermi velocity along direction $x$ in $\left[\mathrm{ms}^{-1}\right]$. The total Fermi velocity is related to the directional Fermi velocity through:

$$
v_{\mathrm{F}}=\sqrt{v_{\mathrm{F}, \mathrm{a}}^{2}+v_{\mathrm{F}, \mathrm{b}}^{2}+v_{\mathrm{F}, \mathrm{c}}^{2}},
$$

where $a, b$, and $c$ represent the three orthogonal crystal directions (fig. 3, left graph).

In this manner, the electron density of states, the Fermi velocity, and the lattice parameters at $\tau=(1.53 \pm 0.08) \times 10^{-14} \mathrm{~S}$ are calculated with $N\left(E_{\mathrm{F}}\right)=15.13 \pm 0.23$ states $/(\mathrm{eV} \times$ unit cell $), v_{\mathrm{F}, \mathrm{x}}=(1.225 \pm 0.008) \times 10^{5} \mathrm{~ms}^{-1}, v_{\mathrm{F}}=(2.121 \pm 0.014) \times 10^{5} \mathrm{~ms}^{-1}$, and $a_{\mathrm{x}}=a_{\mathrm{y}}=a_{\mathrm{z}}=0.5312 \mathrm{~nm}$. Using equation 18 , the critical normal state resistivity $\rho_{\mathrm{c}}$ is calculated at $27.0 \pm 1.4 \mu \Omega \mathrm{cm}$, where $\mathrm{Nb}_{3} \mathrm{Sn}$ is preferentially cubic when $\rho_{\mathrm{n}}>\rho_{\mathrm{c}}$ and preferentially tetragonal when $\rho_{\mathrm{n}}<\rho_{\mathrm{c}}$.

The calculated $\rho_{\mathrm{c}}$ of $27.0 \pm 1.4 \mu \Omega \mathrm{cm}$ is consistent with the critical resistivity $25 \pm 3$ $\mu \Omega \mathrm{cm}$ that is derived from experimental observations, which is an indication of the applicability of the electron lifetime broadening hypothesis (section 2.1).

\subsection{Comparison of calculated $N\left(E_{\mathrm{F}}\right), v_{\mathrm{F}}$, and phonon dispersion curves to literature results}

The normal state resistivity dependent electron density of states $N\left(E_{\mathrm{F}}\right)$ and Fermi velocity $v_{\mathrm{F}}$ were previously derived from experimental data by by Lim et al. [43], Schachinger et al. [16], Orlando et al. [17], and Ghosh et al. [18]. The analyses of Schachinger et al. and Orlando et al. were performed on two samples with different Sn contents, while the analysis of Ghosh et al. was performed on stoichiometric $\mathrm{Nb}_{3} \mathrm{Sn}$ in which disorder was introduced through radiation damage. These analyses utilized various experimental observations, including the heat capacity, the critical temperature, and the upper critical field. 


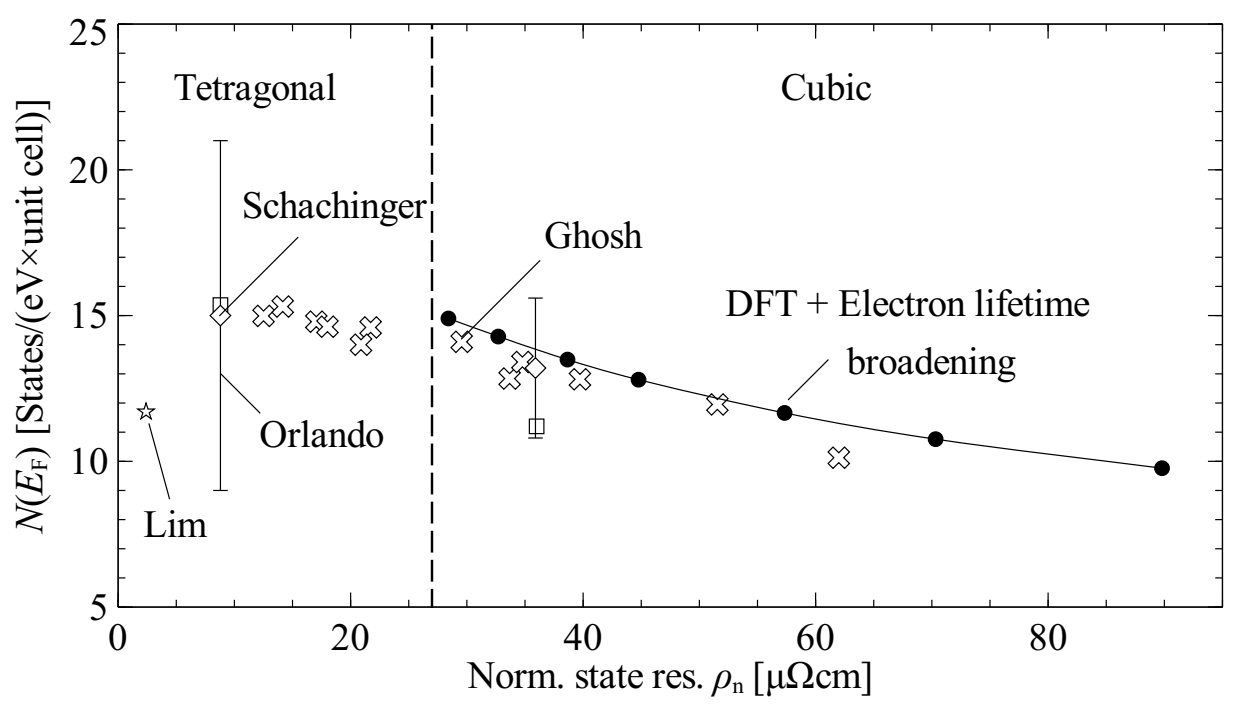

Figure 4. Calculated electron density of states $N\left(E_{\mathrm{F}}\right)$ as a function of $\rho_{\mathrm{n}}$, compared to various literature results from Lim et al. [43], Schachinger et al. [16], Orlando et al. [17], and Ghosh et al. [18].

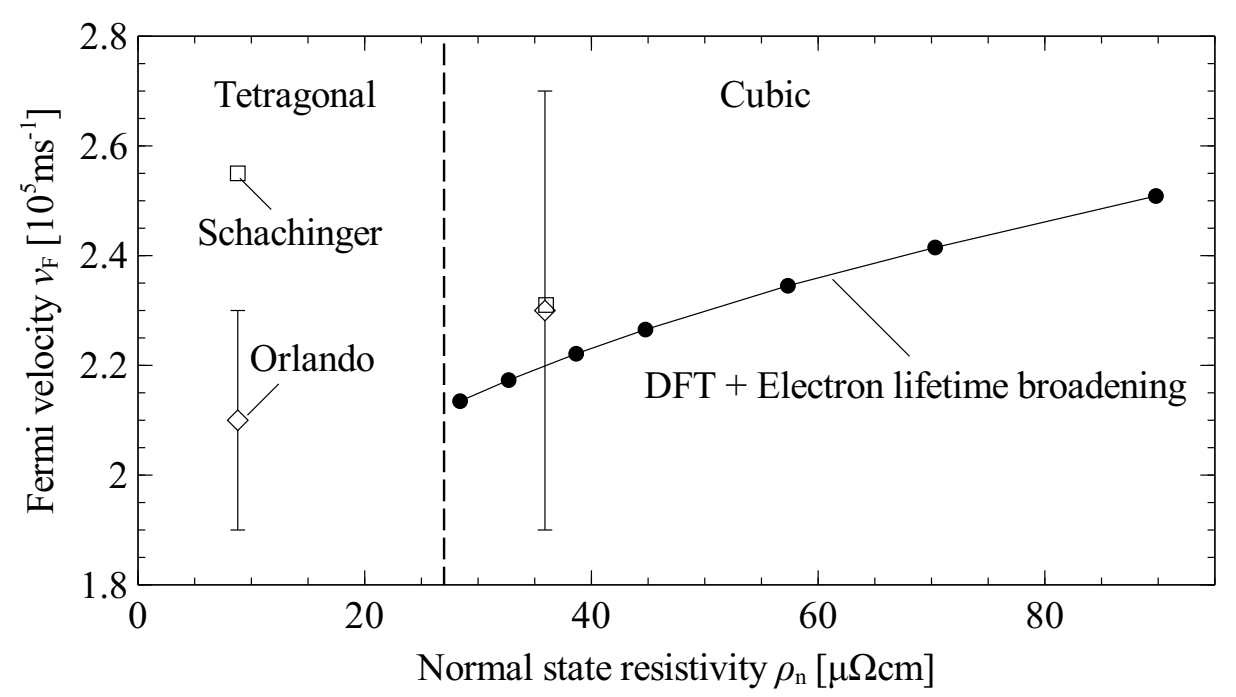

Figure 5. Calculated root mean square Fermi velocity $v_{\mathrm{F}}$ as a function of normal state resistivity $\rho_{\mathrm{n}}$, compared to literature results after Schachinger et al. [16] and Orlando et al. [17].

$\mathrm{Ab}$ initio calculations of the normal state resistivity dependent electron density of states and Fermi velocity are performed and compared to literature results. The results are found to be consistent for both the electron density of states (fig. 4) and the Fermi velocity (fig. 5). The calculations were only performed in the preferentially cubic regime (also see section 3.2). Performing calculations on a single unit cell in the preferentially tetragonal regime gives incorrect results, because this implicitly and incorrectly assumes that the $c$-axis is perfectly aligned throughout the crystal. In reality, it was argued by Kartha et al. [44] and experimentally demonstrated by Goringe et al. 


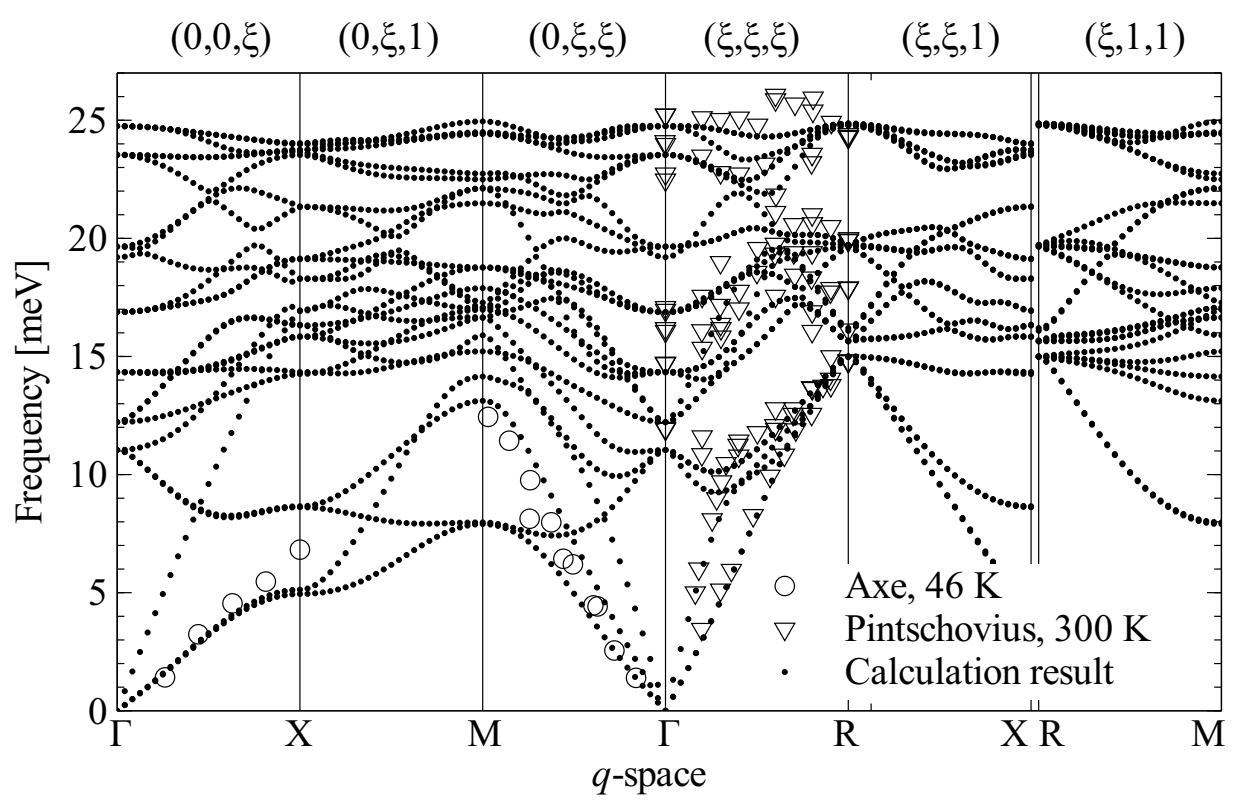

Figure 6. Calculated phonon dispersion curves of disordered $\mathrm{Nb}_{3} \mathrm{Sn}$, compared to experimental observations. The phonon dispersion curves are calculated at $\tau=$ $0.76 \times 10^{-15} \mathrm{~s}$, which corresponds to a normal state resistivity of $57.3 \mu \Omega \mathrm{cm}$. The calculation result is compared to experimental observations by Pintschovious et al. [48] and Axe et al. [47].

[45] and Onozuka et al. [46] in the case of $\mathrm{V}_{3} \mathrm{Si}$, that the martensitic transformation results in tweed modulation. This phenomenon describes the division of the sample into different regions, where the orientation of the short $c$-axis (fig. 3, right graph) varies between the regions. Very fine tweed patterns are observed, with widths as fine as $10 \mathrm{~nm}$ [45], i.e. only one order of magnitude above the lattice constant of the material (see fig. 3, right graph). It is not understood how the formation of the tweed pattern affects the electronic properties nor the local stress and strain state. While this phenomenon might be incorporated in the DFT calculations through the use of a very large supercell, the computational requirements of such a calculation are extreme. This is why the calculations in this paper only consider the properties of preferentially cubic $\mathrm{Nb}_{3} \mathrm{Sn}$.

In fig. 6, calculated phonon dispersion curves are compared to the experimental observations by Axe et al. [47], and Pintschovius et al. [48], while the phonon density of states at three different degrees of disorder is compared to an experimental observation of the frequency dependent phonon density of states, after Schweiss et al. [13] (fig. 7). This comparison is somewhat imperfect, as it compares calculation results of cubic and thus higher-resistivity $\mathrm{Nb}_{3} \mathrm{Sn}$ to experimental results of low-resistivity $\mathrm{Nb}_{3} \mathrm{Sn}$.

In an in-depth discussion of inelastic neutron scattering measurements, Axe et al. [27] identify two mechanisms that contribute to a broadening of the phonon density of states, which are phonon scattering off of thermally excited quasi-particles and direct excitation of quasi-particles across the superconducting gap by phonons. These two 


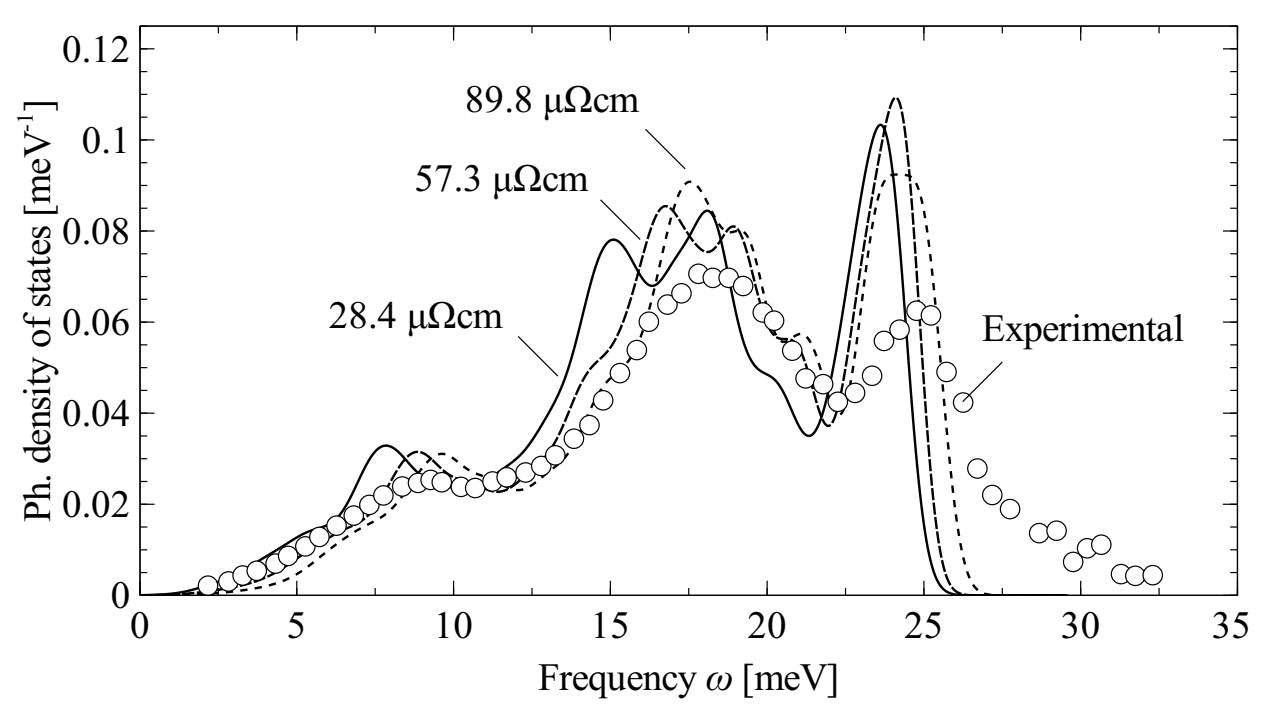

Figure 7. Calculated phonon density of states $F(\omega)$ as a function of vibrational frequency $\omega$, compared to experimental observations. The phonon densities of states are calculated at $\tau=1.45 \times 10^{-15} \mathrm{~s}, 0.76 \times 10^{-15} \mathrm{~s}$, and $0.51 \times 10^{-15} \mathrm{~s}$, which corresponds to $28.4,57.3$, and $89.8 \mu \Omega \mathrm{cm}$ respectively. The experimental result is of low-resistivity $\mathrm{Nb}_{3} \mathrm{Sn}$, after Schweiss et al. [13].

phenomena contribute to a broadening with a full width half max (FWHM) of $1.2 \mathrm{meV}$. To allow for a qualitative comparison of the calculation results and the experimental observations, the calculation results are convoluted with a gaussian function with an fullwidth-at-half-maximum of $1.2 \mathrm{meV}$ (fig. 7). This does not affect the derived electronphonon constant and the characteristic frequency, as these are integrals over the entire frequency range.

Apart from these details, the calculation results are generally very similar to the experimental observations (figures 6 and 7). In general, the calculated frequencies of the various vibrational modes are somewhat low in comparison to the experimental observations, although it is not clear whether this is a small systematic error in the calculation result or due to the imperfect nature of the comparison discussed here.

\subsection{Calculated versus experimentally observed Eliashberg spectra of high-resistivity $\mathrm{Nb}_{3} \mathrm{Sn}$}

To validate whether $\omega_{0}$ and $\alpha_{\text {IM,eff }}^{2}$ are indeed weakly dependent or independent of disorder and thus may be considered as global constants (section 2.2), $\alpha^{2}(\omega) F(\omega)$ of high-resistivity $\mathrm{Nb}_{3} \mathrm{Sn}$ is determined from the calculated electron and phonon density of states through equation 15 and compared to experimental observations of stoichiometric and off-stoichiometric Nb-Sn tunnel junctions by Rudman et al. [8] (fig. 8). Consistent with Rudman's observations, the calculation result indicates that three peaks are present and that the amplitude of $\alpha^{2}(\omega) F(\omega)$ decreases with increasing amount of disorder (i.e. increasing $\left.\rho_{\mathrm{n}}\right)$. The frequencies of the peaks in the calculated spectra are somewhat low frequency in comparison to the experimental result, which could mean that a small 


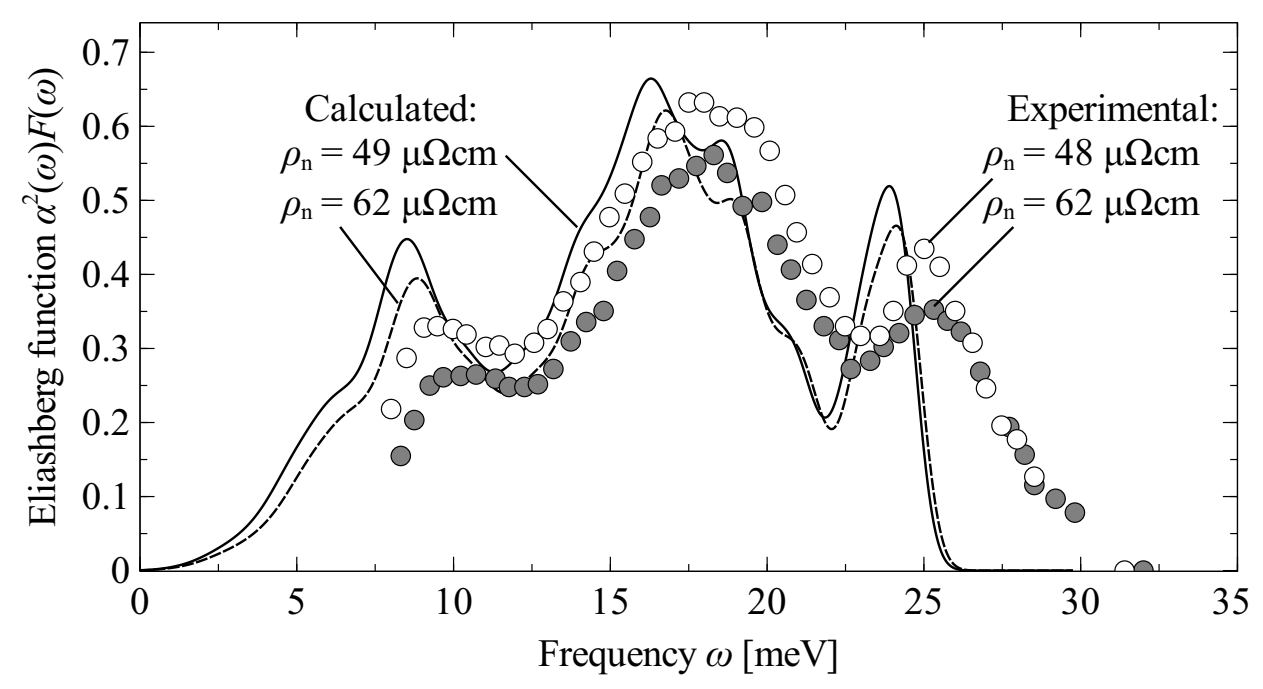

Figure 8. Calculated Eliashberg function $\alpha^{2}(\omega) F(\omega)$ as a function of $\omega$ of disordered $\mathrm{Nb}_{3} \mathrm{Sn}$, compared to $\alpha^{2}(\omega) F(\omega)$ derived from off-stoichiometric tunnel junctions, after Rudman et al. [8].

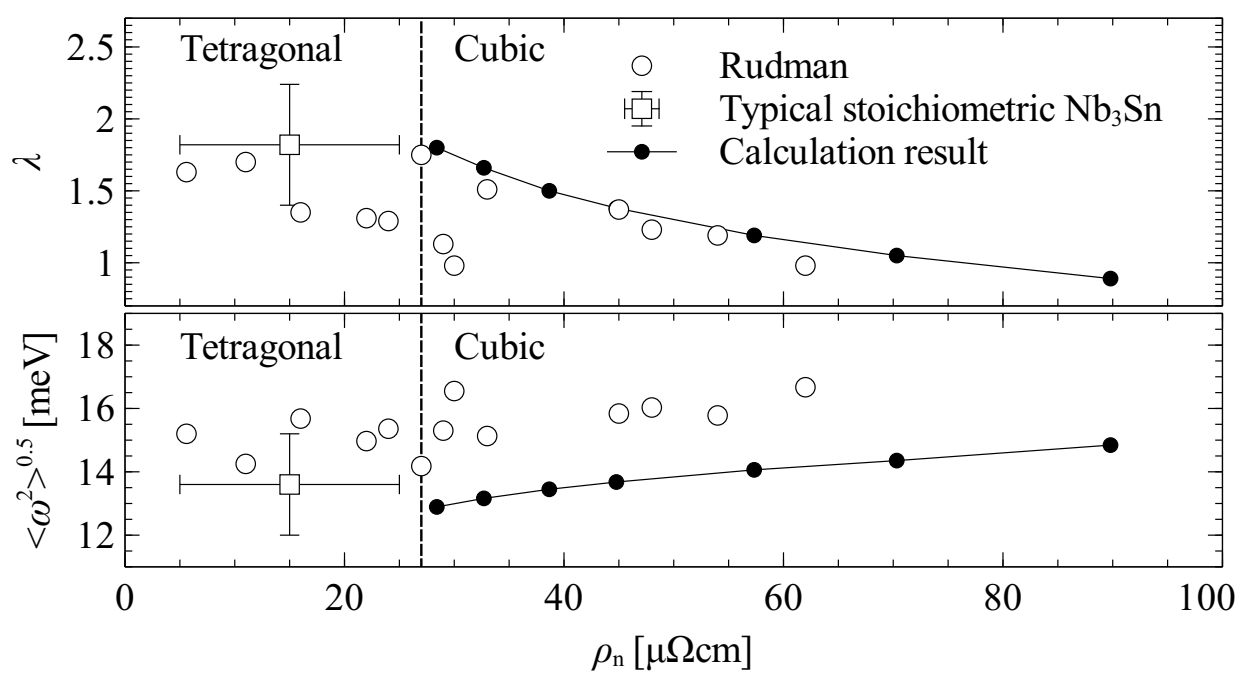

Figure 9. Calculated electron-phonon coupling constant $\lambda$ and characteristic phonon frequency $\left\langle\omega^{2}\right\rangle^{0.5}$ as a function of normal state resistivity $\rho_{\mathrm{n}}$, compared to values derived from experimental observations by Rudman et al. [8]. Also shown are the average literature values and standard deviations of low-resistivity $\mathrm{Nb}_{3} \mathrm{Sn}$, shown in table 1.

systematic error is present in the phonon density of states calculation. However, Rudman et al. [8] points out that some anomalies were observed in the tunnel junction data, which might also imply a slight systematic upward bias in the experimental results.

From the calculated Eliashberg spectra, $\lambda$ and $\left\langle\omega^{2}\right\rangle^{0.5}$ are derived through equations 2 and 6 and compared to experimental observations by Rudman et al. [8] (fig. 9). In addition, from the Eliashberg spectra published by various authors (fig. 1, left graph), $\lambda$ and $\left\langle\omega^{2}\right\rangle^{0.5}$ are derived using equations 2 and 6 (table 1). The 
calculated values of $\lambda$ are very close to Rudman's observations, while the calculated $\left\langle\omega^{2}\right\rangle^{0.5}$ is somewhat lower than Rudman's values over the entire $\rho_{\mathrm{n}}$ range, albeit within one standard deviation of the mean literature values (table 1).

In summary, the calculated electron-phonon coupling constant $\lambda$ is consistent with experimental results over the investigated disorder range. The characteristic frequency $\left\langle\omega^{2}\right\rangle^{0.5}$ is somewhat below the experimentally observed number, but the general trend is consistent. This result indicates that, within the uncertainty of this comparison, it seems reasonable to treat $\alpha_{\mathrm{IM} \text {,eff }}^{2}$ and $\omega_{0}$ as global constants, which supports assumption \#2 (section 2.2).

\section{Comparison of calculation results and experimental observations on the superconducting phase boundary}

\subsection{Critical temperature $T_{\mathrm{c}}$ as a function of normal state resistivity}

Using the expressions for critical temperature given by Kresin (eq. 16) and Allen-Dynes (eq. 17), the normal state resistivity dependent critical temperature is calculated and compared to experimental results (fig. 10).

In this comparison, the emphasis is placed on inductive and heat capacity measurements instead of resistivity measurements. While it is relatively straightforward to produce samples of $\mathrm{Nb}_{3} \mathrm{Sn}$ with very sharp $T_{\mathrm{c}}$ transitions at $T_{\mathrm{c}} \approx 18 \mathrm{~K}$, producing low- $T_{\mathrm{c}} \mathrm{Nb}-\mathrm{Sn}$ samples with sharp $T_{\mathrm{c}}$ transitions is a major challenge. Investigations of the $T_{\mathrm{c}}$ width in low- $T_{\mathrm{c}}$ samples indicate that the $T_{\mathrm{c}}$ transitions are typically several Kelvin wide (see Hellman et al. [49] and Mentink et al. [50]). In resistivity measurements, the measured normal state resistivity may be considered an effective average, but only a fraction of the sample needs to be superconducting in order to form a superconducting path and short the sample. This means that an upward bias in a direct $T_{\mathrm{c}}$ versus $\rho_{n}$ measurement is unavoidable. Inductive and heat capacity measurements do not suffer from this problem so that the latter in particular is considered a 'true' bulk measurement. Unfortunately, the normal state resistivity of samples investigated through inductive and heat capacity measurements is not always known. However, the relationship between normal state resistivity and composition in binary Nb-Sn samples where disorder is not introduced through some other fashion is well understood (Godeke [51], Flükiger [52]), and was empirically summarized by Godeke et al. [51], with:

$$
\rho_{\mathrm{n}}(\beta)=91\left(1-(7 \beta-0.75)^{4}\right)+3.4 \text { for } \beta \leq 25 \%,
$$

where $\beta$ is the atomic tin fraction, and $\rho_{\mathrm{n}}$ is the residual resistivity (i.e. the normal state resistivity slightly above $\left.T_{\mathrm{c}}\right)$ in $[\mu \Omega \mathrm{cm}]$. The uncertainty in this description is estimated to be about $5 \mu \Omega \mathrm{cm}$. Thus, in measurements where the off-stoichiometric composition rather than the normal state resistivity is known, the normal state resistivity is calculated through equation 24.

Besides the resistivity measurements of Orlando et al. [17], in which disorder is introduced through anti-site disorder, aluminum additions, and off-stoichiometry 


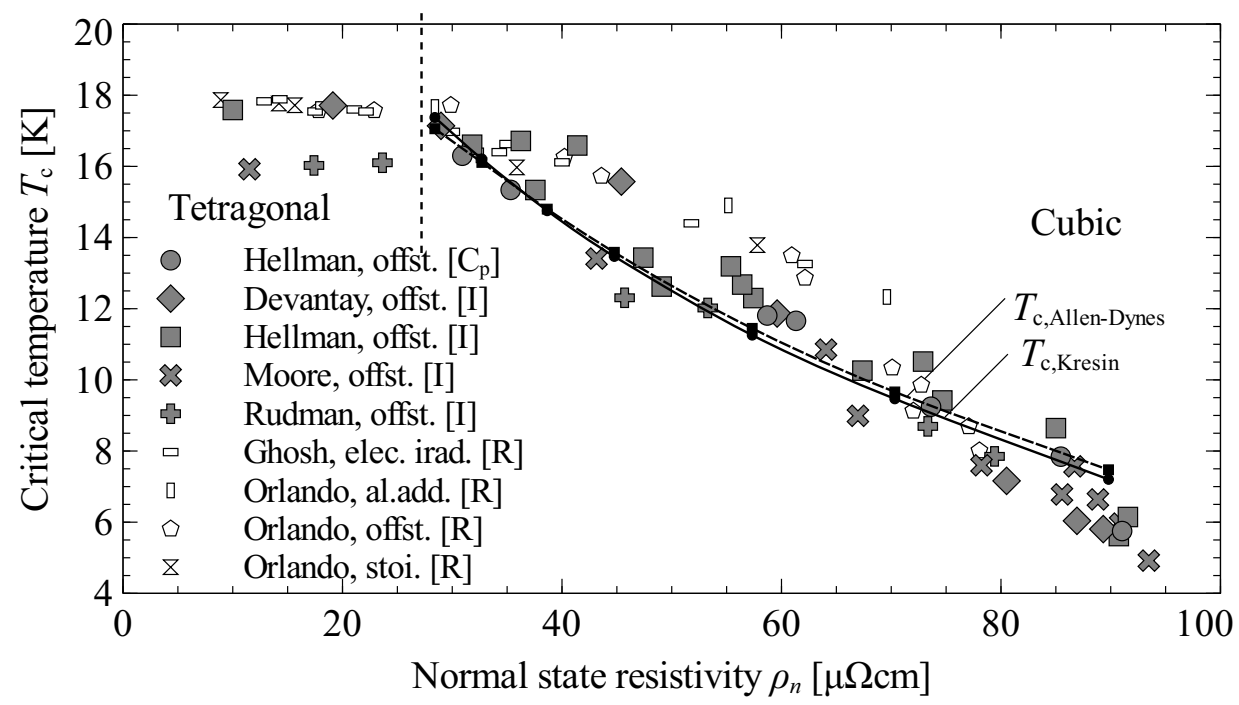

Figure 10. Calculated critical temperature $T_{\mathrm{c}}$ as a function of normal state resistivity $\rho_{\mathrm{n}}$, compared to experimental literature data.

(indicated as 'stoi.', 'al.add', and 'offst.' respectively in fig. 10), and the resistivity measurements of Ghosh et al. [18] in which disorder is introduced through electron irradiation (indicated as 'elec. irad' in fig. 10), various heat capacity and inductive measurements of off-stoichiometric $\mathrm{Nb}-\mathrm{Sn}$ samples are shown, after Hellman et al., Devantay et al. [29], Moore et al. [53], and Rudman et al. [54].

The comparison between the calculation results and experimental observations shows a high degree of consistency, in particular with regards to induce and heat capacity measurements.

\subsection{Upper critical magnetic field $H_{\mathrm{c} 2}$ as a function of critical temperature $T_{\mathrm{c}}$}

In addition to the critical temperature, the upper critical field is investigated. The upper critical magnetic field in phonon-mediated superconductors has been thoroughly investigated in the past, resulting in a number of descriptions. After the initial success of the Ginzburg-Landau-Abrikosov-Gor'kov (GLAG) theory [55, 56, 57], various corrections were considered and developed to account for the different phenomena which affect the upper critical magnetic field. The temperature dependence of $H_{\mathrm{c} 2}$ was described by Maki and De Gennes [58], under the assumption of a dirty superconductor, a spherical Fermi surface, a weakly energy dependent electronic density of states, and a weak-coupling interaction. Subsequent refinements were made to include various effects, such as the impurity dependence of $H_{\mathrm{c} 2}$ by Helfand et al. [59, 60], Fermi surface anisotropy by Hohenberg et al. [61] and Schachinger et al. [16], spin-orbit coupling by Werthamer et al. [62], Schopohl et al. [63], and Rieck et al. [64], anisotropic scattering by Schopohl et al. [65], Pauli paramagnetic limiting by Orlando et al. [17, 66] and Rieck et al. [64], strong coupling effects by Werthamer et al. [67], Masharov [68], and Schossmann et al. [69], p- and d-wave scattering by Rieck at al. [64] and the energy- 
dependence of $N\left(E_{\mathrm{F}}\right)$ by Schossmann et al. [70]. A useful overview of these various mechanisms is provided by Rieck et al. [64].

A number of authors investigated the temperature dependence of the upper critical magnetic field, in an attempt to determine which of the aforementioned phenomena should be considered to get a realistic description of $\mathrm{Nb}_{3} \mathrm{Sn}$. Orlando et al. [17] considered the influence of Pauli paramagnetic limiting and spin-orbit coupling and found that a large amount of spin-orbit coupling was required to counteract the reduction of $H_{\mathrm{c} 2}(0)$ due to Pauli paramagnetic limiting. However, in a subsequent paper by the same authors [66], it was pointed out that the original paper incorrectly assumed a first-order transition rather than a second-order transition, resulting in an incorrect description of the Pauli limiting field. Schachinger et al. [16] used a description which emphasized Pauli paramagnetic limiting and Fermi surface anisotropy, without considering spin-orbit coupling, and investigated the same experimental data that was obtained by Orlando et al. [17]. In this approach, a free parameter was used to describe the extent by which Fermi anisotropy raises $H_{\mathrm{c} 2}(0)$.

Rieck et al. constructed a highly comprehensive model that takes into account various phenomena, such as Fermi surface anisotropy, Pauli limiting, s-, p- and d-wave scattering, and spin-orbit coupling where the magnitude of each of these phenomena is described with a free parameter. In evaluating the same experimental data that Orlando et al. [17] obtained, Rieck et al. determined that the experimental data was accurately described with dirty limit theory in the case of the off-stoichiometric sample and clean limit theory in the case of the low-resistivity stoichiometric sample, and that allowing for fitting of the various free parameters which account for the influence of the other physical phenomena only led to a minor improvement in fitting accuracy. In other words, the influence of the various phenomena is minor or the influences of the various phenomena cancel each other out.

Consistent with this analysis, it was subsequently demonstrated by Godeke et al. [71] that the Maki-De Gennes description (which is a dirty limit, weak coupling approximation that does not consider other phenomena such as Pauli limiting and spinorbit coupling) matches the observed temperature dependence of $H_{\mathrm{c} 2}$ in a large variety of binary and ternary $\mathrm{Nb}_{3} \mathrm{Sn}$ wires, bulk samples, and thin films.

It should be emphasized that most of these analyses mainly concern themselves with the experimentally-determined temperature-dependent upper critical field. Here, however, $H_{\mathrm{c} 2}$ at $0 \mathrm{~K}$ is calculated from other microscopic parameters such as the calculated Fermi velocity.

Fig. 11 shows a collection of experimental observations of $H_{\mathrm{c} 2}(0)$ as a function of $T_{\mathrm{c}}$ in binary and ternary $\mathrm{Nb}_{3} \mathrm{Sn}$ samples. Unlike the $T_{\mathrm{c}}\left(\rho_{\mathrm{n}}\right)$ dependence (fig. 10), where the use of resistively determined $T_{\mathrm{c}}$ as a function of $\rho_{\mathrm{n}}$ measurements can lead to incorrect results, resistance measurements can be used to determine the $H_{\mathrm{c} 2}(0)$ as a function of $T_{\mathrm{c}}$ relationship, because in the preferentially cubic regime, $\mu_{0} H_{\mathrm{c} 2}(0)$ rises with increasing $T_{\mathrm{c}}$ and thus a resistive measurement of $H_{\mathrm{c} 2}(T)$ probes the same compositional fraction inside a sample as a resistive measurement of $T_{\mathrm{c}}$. 
The experimental observations (fig. 11) consist of resistive measurements of thin films, bulk samples, and strands, after Mentink et al. [50, 72], Zhou et al. [30], Jewell et al. [73], Orlando et al. [2], Devantay et al. [29], and Godeke et al. [71]. In addition, radio-frequency measurements of bulk material were taken by Foner et al. [74], and magnetic measurements of bulk material were taken by Arko et al. [25] and Jewell et al. [73]. Critical current density measurements of binary thin film samples were performed by Mentink et al. [3]. Magnetic measurements of $\mathrm{Nb}_{3} \mathrm{Sn}$ wires were taken by Naus et al. [75], in which $T_{\mathrm{c}}$ and the Kramer extrapolated $H_{\mathrm{K}}(4.2 \mathrm{~K})$ were determined. From these measurements, $H_{\mathrm{c} 2}(0)$ was extrapolated through a method that is discussed elsewhere [50].

Here, the upper critical field is calculated in the dirty to intermediate limit, where strong-coupling corrections and Pauli limiting are accounted for. The influence of these phenomena is chosen because they are well-described in literature and don't require the use of free parameters. The accuracy of the calculations may potentially be enhanced further, which is discussed in section (5).

The slope of $\delta H_{\mathrm{c} 2}^{+} / \delta T$ at $0 \mathrm{~T}$ is described by Ginzburg-Landau-Abrikosov-Gor'kov (GLAG) theory $[5,17,55,56,57]$, with:

$$
\left.\frac{-\delta\left(\mu_{0} H_{\mathrm{c} 2}\right)}{\delta T}\right|_{T_{\mathrm{c}}}=\left(\frac{24 \pi^{2} k_{\mathrm{B}}^{2}}{7 \zeta(3) \hbar e}\right) \frac{\eta_{\mathrm{H}_{\mathrm{c} 2} T_{\mathrm{c}}(1+\lambda)^{2}}}{v_{\mathrm{F}}^{2} X(Z)},
$$

where $\delta\left(\mu_{0} H_{\mathrm{c} 2}\right) / \delta T$ is in $[\mathrm{T} / \mathrm{K}], \zeta(3)$ is Apery's constant. $\eta_{\mathrm{Hc} 2}$ is the strong coupling correction factor for $\mu_{0} H_{\mathrm{c} 2}$ [68]. $Z$ is the reduced collision frequency [59], which is closely related to mean scattering time:

$$
Z=\frac{\hbar}{2 \pi k_{\mathrm{B}} T_{\mathrm{c}}(1+\lambda) \tau}=\frac{\hbar v_{\mathrm{F}}}{2 \pi k_{\mathrm{B}} T_{\mathrm{c}}(1+\lambda) l_{\mathrm{mfp}}} .
$$

Here $l_{\mathrm{mfp}}$ is the mean free path. $X(Z)$ is the Gor'kov function [57], with:

$$
X(Z)=\frac{\sum_{v=0}^{\infty}(2 v+1)^{-2}(2 v+1+Z)^{-1}}{\sum_{v=0}^{\infty}(2 v+1)^{-3}} .
$$

The upper critical field $H_{\mathrm{c} 2}^{+}$is calculated from $\delta\left(H_{\mathrm{c} 2}\right) / \delta T$ using parameter $h_{\mathrm{c} 2}^{*}$, with:

$$
H_{\mathrm{c} 2}^{+}(0)=\left.h_{\mathrm{c} 2}^{*}(Z) \frac{-\delta\left(H_{\mathrm{c} 2}\right)}{\delta T}\right|_{T_{\mathrm{c}}} T
$$

Combining equations 25 and 28, the upper critical magnetic field without Pauli limiting $H_{\mathrm{c} 2}^{+}$is expressed as:

$$
\mu_{0} H_{\mathrm{c} 2}^{+}(0)=\eta_{\mathrm{Hc} 2} X(Z)^{-1} h_{\mathrm{c} 2}^{*}(Z)\left(\frac{24 \pi^{2} k_{\mathrm{B}}^{2}}{7 \zeta(3) \hbar e}\right) \frac{T_{\mathrm{c}}^{2}(1+\lambda)^{2}}{v_{\mathrm{F}}^{2}} .
$$


According to Masharov et al. [68] the strong coupling correction factor $\eta_{\mathrm{Hc} 2}$ is expressed as:

$$
\eta_{H_{\mathrm{c} 2}}=1+\left(\frac{\pi k_{\mathrm{B}} T_{\mathrm{c}}}{\left\langle\omega^{2}\right\rangle^{0.5}}\right)\left(0.6 \ln \left(\frac{\left\langle\omega^{2}\right\rangle^{0.5}}{k_{\mathrm{B}} T_{\mathrm{c}}}\right)-0.26\right),
$$

where $k_{\mathrm{B}} T_{\mathrm{c}}$ is in $[\mathrm{eV}],\left\langle\omega^{2}\right\rangle^{0.5}$ is in $[\mathrm{eV}]$, and $\eta_{\mathrm{Hc} 2}$ is a dimensionless parameter. At $T_{\mathrm{c}}$ $=17.2 \mathrm{~K}$ (i.e. $\left.\rho_{\mathrm{n}}=28.4 \mu \Omega \mathrm{cm}\right), \eta_{\mathrm{Hc} 2}$ is calculated at 1.13 , while at $T_{\mathrm{c}}=7.3 \mathrm{~K}$ (i.e. $\rho_{\mathrm{n}}$ $=90 \mu \Omega \mathrm{cm})$, it is calculated at 1.03. The $Z$ dependent $h_{\mathrm{c} 2}^{*}$ is taken from Rieck et al. [64]. This function is equal to the dirty limit value of 0.69267 over most of the disorder range, but it rises to 0.72 near the clean limit. The $Z$ dependent $X$ is calculated through equation 27. The result is linearized between $Z=1.56$ to $Z=17.5$ :

$$
X(Z)^{-1} h_{\mathrm{c} 2}^{*}(Z) \approx 0.608 Z+0.766
$$

which results in an average deviation of $0.4 \%$ and a maximum deviation of $0.6 \%$ between the exact and linearized expressions in the range of $Z=1.56$ to $17.5 . H_{\mathrm{c} 2}^{+}(0)$ is then expressed as:

$$
\begin{aligned}
& \mu_{0} H_{\mathrm{c} 2}^{+}(0)=\eta_{\mathrm{Hc} 2}\left(C_{\mathrm{O} 1} \frac{T_{\mathrm{c}}^{2}(1+\lambda)^{2}}{v_{\mathrm{F}}^{2}}+C_{\mathrm{O} 2} \frac{T_{\mathrm{c}}(1+\lambda)}{v_{\mathrm{F}} l_{\mathrm{mfp}}}\right), \\
& C_{\mathrm{O} 1}=\frac{0.766 \times 24 \pi^{2} k_{\mathrm{B}}^{2}}{7 \zeta(3) \hbar e}=2.43 \times 10^{8}\left[\mathrm{Tm}^{2} \mathrm{~K}^{-2} \mathrm{~s}^{-2}\right], \\
& C_{\mathrm{O} 2}=\frac{0.608 \times 12 \pi k_{\mathrm{B}}}{7 \zeta(3) e}=2.35 \times 10^{-4}\left[\mathrm{Tm}^{2} \mathrm{~K}^{-1} \mathrm{~s}^{-1}\right] .
\end{aligned}
$$

After Orlando et al. [66], the Pauli paramagnetic limiting field in the absence of spinorbit coupling is expressed as:

$$
\mu_{0} H_{P}(0)=1.858 \times T_{\mathrm{c}}(1+\lambda),
$$

where $T_{\mathrm{c}}$ is in $[\mathrm{K}]$ and $\mu_{0} H_{\mathrm{P}}$ is in $[\mathrm{T}]$. Following Orlando et al. [66], the expression for $H_{\mathrm{c} 2}(0)$ is:

$$
\left(H_{\mathrm{c} 2}(0)\right)^{-2}=\left(H_{\mathrm{c} 2}^{+}(0)\right)^{-2}+2\left(H_{\mathrm{P}}(0)\right)^{-2} .
$$

Relative to the $H_{\mathrm{c} 2}^{+}(0)$, Pauli limiting reduces $H_{\mathrm{c} 2}(0)$ by $13 \%$ at $T_{\mathrm{c}}=17.2 \mathrm{~K}$ (i.e. $\rho_{\mathrm{n}}=$ $28.4 \mu \Omega \mathrm{cm}$ ), and $17 \%$ at $T_{\mathrm{c}}=7.3 \mathrm{~K}$ (i.e. $\rho_{\mathrm{n}}=90 \mu \Omega \mathrm{cm}$ ).

The final result is shown in figure 11. It is clear that the calculated results are generally consistent with experimental observations, albeit somewhat on the low end of the experimental range, in particular in the $T_{\mathrm{c}}$ regime of 14 to $16 \mathrm{~K}$. 


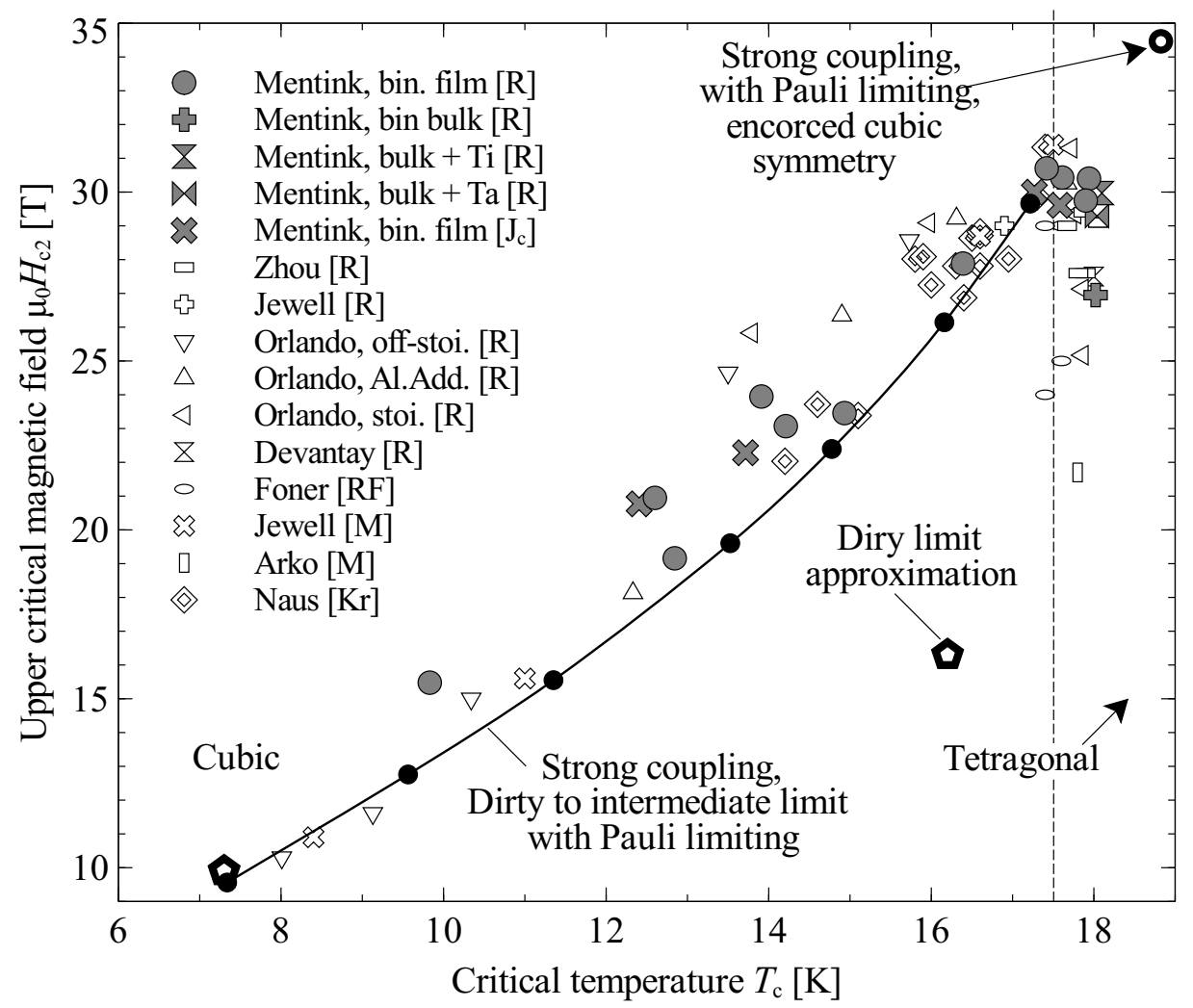

Figure 11. Calculated upper critical field $\mu_{0} H_{\mathrm{c} 2}(0)$ as a function of critical temperature $T_{\mathrm{c}}$, compared to literature data.

\subsection{Effect of the martensitic transformation on $T_{\mathrm{c}}$ and $\mu_{0} H_{\mathrm{c} 2}(0)$}

In fig. 11 a significant drop in $\mu_{0} H_{\mathrm{c} 2}(0)$ is observed near $T_{\mathrm{c}}=18 \mathrm{~K}$, that seems to correlate with the onset of the tetragonal regime. Here we argue that these two phenomena are related.

To investigate this possibility, a calculation is performed where the martensitic transformation is suppressed, i.e. rather than allowing the unit cell to find the optimal (non-cubic) configuration, cubic symmetry is enforced and the properties of the crystal are evaluated. Unfortunately, only the electronic properties of this unstable crystal can be evaluated, as the unstable nature prevents calculation of the vibrational properties. Instead, the phonon density of states at $\tau=1.45 \times 10^{-14} \mathrm{~s}$, corresponding to $\rho_{\mathrm{n}}=$ $26.7 \mu \Omega \mathrm{cm}$, is taken. At $\tau=1.91 \times 10^{-14} \mathrm{~s}$, corresponding to $\rho_{\mathrm{n}}=21.4 \mu \Omega \mathrm{cm}$, a critical temperature of $18.8 \mathrm{~K}$ and an upper critical field of $34.5 \mathrm{~T}$ is found, thus implying that if the tetragonal transformation does not occur, the superconducting properties continue to rise with decreasing normal state resistivity.

This statement is controversial and in past publications two arguments were given for to explain why the occurrence of the martensitic transformation does not result in the reduction of $\mu_{0} H_{\mathrm{c} 2}$ :

First of all, it is commonly assumed that $\mathrm{Nb}_{3} \mathrm{Sn}$ may be approximated as a dirty limit superconductor. This means that in equation 26, $Z$ may be approximated at 
$\infty$ and $X(Z)$ may be approximated at $0.853 \times Z$. Also ignored strong coupling effects and Pauli limiting, the upper critical field may then be related to the electronic heat capacity, so that a rather simple expression of $\mu_{0} H_{\mathrm{c} 2}(0)$ may be deduced:

$$
\begin{aligned}
& \gamma^{*}=\frac{\pi^{2} k_{\mathrm{B}}^{2} N\left(E_{\mathrm{F}}\right)(1+\lambda)}{3 e}, \\
& \mu_{0} H_{\mathrm{c} 2 \text {,dirty }}(0)=C_{\text {dirty }} T_{\mathrm{c}} \gamma^{*} \rho_{\mathrm{n}}, \\
& C_{\text {dirty }}=3.11 \times 10^{3}\left[\mathrm{TKm}^{2} \mathrm{~J}^{-1} \Omega^{-1}\right] .
\end{aligned}
$$

where $\gamma^{*}$ is the renormalized electronic heat capacity in $\left[\mathrm{JK}^{-2} \mathrm{~m}^{-3}\right]$ (see, for instance, analyses by Orlando et al. [17] and Guritanu et al. [33]). Thus, if this equation is applicable towards the clean limit, then $\mu_{0} H_{\mathrm{c} 2}(0)$ is proportional to the normal state resistivity, so that one may speculate that the drop in $\mu_{0} H_{\mathrm{c} 2}(0)$ is due to the drop in $\rho_{\mathrm{n}}$. A problem with this expression is that $\mathrm{Nb}_{3} \mathrm{Sn}$ is a intrinsic type II superconductor, which means that it exhibits type-II behavior without being in the dirty limit (see for instance Fetter and Hohenberg [76]). Indeed, while the calculation result matches the experimental in the dirty limit (at $\left.T_{\mathrm{c}}=7.2 \mathrm{~K}\right), \mu_{0} H_{\mathrm{c} 2}(0)=16.2 \mathrm{~T}$ is found at $T_{\mathrm{c}}=16.2 \mathrm{~K}$ which is highly inconsistent with the experimental data (fig. 11). Given the inapplicability of equation 37 towards the clean limit, as well as the fact that $\mu_{0} H_{\mathrm{c} 2}(0)$ is no longer proportional to $\rho_{\mathrm{n}}$ when the material is not in the dirty limit, this argument appears to be incorrect.

A more recent argument is given by Zhou et al. [30], in which a detailed experimental investigation was undertaken to show that Nb-Sn samples may contain tetragonal $\mathrm{Nb}_{3} \mathrm{Sn}$ while simultaneously having an upper critical field of $29 \mathrm{~T}$, as determined by resistive measurements. However, after a subsequent thorough investigation which considered the possibility of minor compositional inhomogeneity in the samples, it was concluded that the observed $\mu_{0} H_{\mathrm{c} 2}(0)$ of $29 \mathrm{~T}$ is likely due to a small fraction of cubic Nb-Sn in the samples, and that the drop in $\mu_{0} H_{\mathrm{c} 2}(0)$ does coincide with the occurrence of tetragonal $\mathrm{Nb}_{3} \mathrm{Sn}$ [77].

\section{Discussion}

One of the starting assumptions taken in this paper is that disorder is a major determinant for the occurrence of the martensitic transformation, $T_{\mathrm{c}}$ and $H_{\mathrm{c} 2}(0)$, regardless whether disorder is introduced through anti-site disorder, radiation damage, or off-stoichiometry, as was previously argued by Orlando et al. [2]. The consistency between the various calculation results and experimental observations is a strong indication that this assumption is indeed reasonable.

One could further speculate that disorder uniquely determines the microscopic properties (i.e. $N\left(E_{\mathrm{F}}\right), v_{\mathrm{F}}, F(\omega), l_{\mathrm{mfp}}$ ) of $\mathrm{Nb}_{3} \mathrm{Sn}$, regardless of the manner in which disorder is introduced. The validity of this statement was investigated by performing supercell calculations at various niobium to tin ratios for a given $\tau$. The results of this investigation indicate that the presence of excess niobium results in a reduced $N\left(E_{\mathrm{F}}\right)$ 
even when $\tau$ is fixed. For instance, fixing $\tau$ at $1.27 \times 10^{-14} \mathrm{~s}$ and comparing supercells with $25,23.4$ and 21.9 at.\% $\mathrm{Sn}, N\left(E_{\mathrm{F}}\right)=14.1,11.2$, and 9.7 states / $(\mathrm{eV} \times$ unit cell) are found, respectively. Thus, when off-stoichiometry is considered, $\tau$ does not uniquely determine the microscopic properties and electron-lifetime broadening should be considered a rather poor proxy for off-stoichiometry. The strong link between $\rho_{\mathrm{n}}, T_{\mathrm{c}}$ and $\mu_{0} H_{\mathrm{c} 2}(0)$ as observed by Orlando et al. [2] may be attributed to the fact that $\rho_{\mathrm{n}}$ is inversely proportional to $N\left(E_{\mathrm{F}}\right), T_{\mathrm{c}}$ is roughly proportional to $\lambda$ and thus to $N\left(E_{\mathrm{F}}\right)$, and $\mu_{0} H_{\mathrm{c} 2}(0)$ is strongly determined by $T_{\mathrm{c}}$. Noting that $T_{\mathrm{c}}$ versus $\rho_{\mathrm{n}}$ is roughly inversely proportional to $\rho_{\mathrm{n}}$ (fig. 10) it is clear that a reduction in $N\left(E_{\mathrm{F}}\right)$ due to off-stoichiometry leads to a similar $T_{\mathrm{c}}$ versus $\rho_{\mathrm{n}}$ dependency as a reduction in $N\left(E_{\mathrm{F}}\right)$ resulting from an increase in $\tau$. In summary, introducing an electron-lifetime broadening assumption to investigate disorder is a reasonable approach for investigating the influence of disorder in general, but further research is needed to distinguish between different types of disorder, for instance anti-site disorder and off-stoichiometry.

In this paper, the effective Coulomb repulsion term is treated as a constant equal to 0.125. This statement is based on experimental evidence, for instance the experimentally observations of $\mu^{*}$ by Rudman in a series of $\mathrm{Nb}_{3} \mathrm{Sn}$ samples over a wide disorder range (Fig. 2). In the paper by Bennemann and Garland [78] two different estimates are provided for transition metals, yielding 0.2 and 0.17 for $\mathrm{Nb}_{3} \mathrm{Sn}$ with a critical temperature of $18 \mathrm{~K}$. Conversely, in other papers the coupling constant is commonly held at about 0.1 for $\mathrm{Nb}_{3} \mathrm{Sn}$ over a wide disorder range [17, 19, 20]. Given this uncertainty in the literature and the availability of experimental data by Rudman (Fig. 2), the constant $\mu^{*}$ used here seems reasonable. To illustrate, an uncertainty in $\mu^{*}$ of 0.02 corresponds to a $T_{\mathrm{c}}$ uncertainty of about $1 \mathrm{~K}$ over the entire disorder range.

The calculated $\mu_{0} H_{\mathrm{c} 2}(0)$ versus $T_{\mathrm{c}}$ curve in fig. 11 is generally consistent with the experimental data within the scatter of the experimental data. Previous published investigations and reviews identify a host of potential phenomena that may impact $\mu_{0} H_{\mathrm{c} 2}(0)$ (see section 4.2). For instance, for a given $T_{\mathrm{c}}, \mu_{0} H_{\mathrm{c} 2}(0)$ may be further increased due to Fermi surface anisotropy as described by Schachinger et al. [16], leading to a reported $5 \%$ increase (i.e. another $1.3 \mathrm{~T}$ ) in $\mu_{0} H_{\mathrm{c} 2}(0)$ at $T_{\mathrm{c}}=16 \mathrm{~K}$. It is clear from fig. 11 that incorporating this phenomenon would indeed result in greater overlap between the experimental and calculated $\mu_{0} H_{\mathrm{c} 2}(0)$. A downside of Schachinger's description and other similar descriptions is that it does not directly relate the degree of increase in $\mu_{0} H_{\mathrm{c} 2}(0)$ to properties of the Fermi surface but rather relies on free parameters. This is undesirable as it affects the reliability of the calculations, and as such, a less accurate description of the upper critical field is used here.

\section{Conclusions}

A detailed investigation is performed to investigate the effects of disorder on the normal state and superconducting properties of $\mathrm{Nb}_{3} \mathrm{Sn}$. This investigation combines firstprinciples calculations with microscopic theory. A comprehensive literature review is 
performed to accomplish this goal

Evaluated properties include the occurrence of the martensitic transformation, the electron density of states, the Fermi velocity, the phonon density of states, the Eliashberg spectrum, the critical temperature, and the upper critical magnetic field as a function of disorder. This investigation provides a comprehensive review of how $\mathrm{Nb}_{3} \mathrm{Sn}$ may be understood in terms of microscopic theory.

The calculation results are validated through comparisons to experimental observations as well as previously published calculation results. An excellent degree of consistency is demonstrated.

\section{Acknowledgements}

This work was supported in part by the Director, Office of Science, High Energy Physics, Basic Energy Sciences, the U.S. Department of Energy under Contract DEAC02-05CH11231. The DFT and DFPT calculations were performed using computing resources from the Cornell NanoScale Science and Technology Facility and the National Energy Research Scientific Computing Center, with special thanks to Derek Stewart and Robert Ryne.

[1] B. T. Matthias, T. H. Geballe, S. Geller, and E. Corenzwit, "Superconductivity of $\mathrm{Nb}_{3} \mathrm{Sn}$ ", Phys. Rev. 95, 1435-1435 (1954).

[2] T. P. Orlando, J. A. Alexander, S. J. Bending, J. Kwo, S. J. Poon, R. H. Hammond, M. R. Beasley, E. J. McNiff, and S. Foner, "The role of disorder in maximizing the upper critical field in the Nb-Sn system", IEEE Trans. on Magn. 17, 368 - 369 (1981).

[3] M. G. T. Mentink, "An Experimental and Computation Study of Strain Sensitivity in Superconducting $\mathrm{Nb}_{3} \mathrm{Sn}$ ", $\mathrm{PhD}$ thesis, University of Twente (2014).

[4] L. R. Testardi, and L. F. Mattheiss, "Electron Lifetime Effects on Properties of A15 and bcc Materials", Phys. Rev. Lett. 41, 1612 - 1615 (1978).

[5] L. F. Mattheiss, and L. R. Testardi, "Electron-lifetime effects on properties of $\mathrm{Nb}_{3} \mathrm{Sn}, \mathrm{Nb}_{3} \mathrm{Ge}$, and Ti-V-Cr alloys", Phys. Rev. B, 20, 2196 - 2200 (1979).

[6] G. M. Eliashberg, Zh. Eksp. Teor. Fiz. 38, 966 (1960).

[7] W. D. Markiewicz, "Elastic stiffness model for the critical temperature $T_{\mathrm{c}}$ of $\mathrm{Nb}_{3} \mathrm{Sn}$ including strain dependence", Cryog. 44, 767 - 782 (2004).

[8] D. A. Rudman and M. R. Beasley, "Microscopic superconducting parameters from tunneling in A15 Nb-Sn", Phys. Rev. B 30, 2590 - 2594 (1984).

[9] L. Y. L. Shen, "Tunneling into a High- $T_{\mathrm{c}}$ Superconductor-Nb ${ }_{3}$ Sn", Phys. Rev. Lett. 29, 1082 - 1086 (1972).

[10] J. Geerk, U. Kaufmann, W. Bangert, and H. Rietschel, "Electron tunneling into $\mathrm{Nb}_{3} \mathrm{Sn}_{2} \mathrm{Nb}_{3} \mathrm{Ge}$, and $\mathrm{Nb}_{3} \mathrm{Al}$ ", Phys. Rev. B 33, 1621 - 1626 (1986).

[11] J. K. Freericks, A. Y. Liu, and A. Quandt, "Nonconstant electronic density of states tunneling inversion of A15 superconductors: $\mathrm{Nb}_{3}$ Sn", Phys. Rev. B 64, 224510 (2002).

[12] E. L. Wolf, "Electron tunneling spectroscopy", Rep. Prog. Phys. 41, 1439 - 1508 (1978).

[13] B. P. Schweiss, B. Renker, E. Schneider, and W. Reichardt, "Phonon Spectra of A-15 Compounds and Ternary Molybdenum Chalcogenides", Superc. in d- and f-band met., 189-208 (1976).

[14] W. L. McMillan, "Transition Temperature of Strong-Coupled Superconductors", Phys. Rev. 167, nr. 2, 331 - 344 (1968). 
[15] J. J. Hopfield, "Angular momentum and transition-metal superconductivity", Phys. Rev. 186, vol. 186, nr. 2, 443 - 451 (1969).

[16] E. Schachinger and M. Prohammer, "Anisotropy effects in the A-15 superconductor $\mathrm{Nb}_{3} \mathrm{Sn}$ ", Physica C 156, 701 - 706 (1988).

[17] T. P. Orlando, E. J. McNiff Jr., S. Foner, and M. R. Beasley, "Critical fields, Pauli paramagnetic limiting, and material parameters of $\mathrm{Nb}_{3} \mathrm{Sn}$ and $\mathrm{V}_{3} \mathrm{Si}$ ", Phys. Rev. B 19, 4545 - 4561 (1979).

[18] A. K. Ghosh, M. Gurvich, H. Wiesmann, and Myron Strongin, "Density of states in two A-15 materials", Phys. Rev. B, 18, 6116 - 6121 (1978).

[19] V. Z. Kresin, "On the critical temperature for any strength of the electron-phonon coupling", Phys. Lett. A 122, 434 - 438 (1987).

[20] P. B. Allen and R. C. Dynes, "Transition temperature of strong-coupled superconductors reanalyzed", Phys. Rev. B 12, 905 - 922 (1975).

[21] P. Giannozzi et al., "Quantum Espresso: a modular and open-source software project for quantum simulations of materials", J. Phys. Condens. Matter. 21, 395502 (2009).

[22] J. P. Perdew, J. A. Chevary, S. H. Vosko, K. A. Jackson, M. R. Pederson, D. J. Singh, and C. Fiolhais, "Atoms, molecules, solids, and surfaces: Applications of the generalized gradient approximation for exchange and correlation", Phys. Rev. B. 46, 6671 - 6687 (1992).

[23] Quantum Espresso website: http://www.quantum-espresso.org/

[24] R. Mailfert, B. W. Batterman, and J. J. Hanak, "Low Temperature Structure Transformation in $\mathrm{Nb}_{3} \mathrm{Sn}^{\prime}$, Phys. Lett. 24A, 315-316 (1967).

[25] A. J. Arko, D. H. Lowndes, F. A. Muller, L. W. Roeland, J. Wolfrat, A. T. van Kessel, H. W. Myron, F. M. Mueller, and G. W. Webb, "De Haas-van Alphen Effect in the High- $T_{\mathrm{c}}$ A15 Superconductors $\mathrm{Nb}_{3} \mathrm{Sn}$ and $\mathrm{V}_{3} \mathrm{Si}$ ", Phys. Rev. Lett. 40, 1590 - 1593 (1978).

[26] Y. Watanabe, N. Toyota, T. Inoue, H. Komatsu, and H. Iwasaki, "An X-ray Diffraction Study of Martensitic Transformation in Ti-Doped $\mathrm{Nb}_{3}$ Sn", Jap. J. of Appl. Phys. 27, 2218-2223 (1988).

[27] J. D. Axe and G. Shirane, "Inelastic-neutron-scattering study of acoustic phonons in $\mathrm{Nb}_{3} \mathrm{Sn}$ ", Phys. Rev. B. 8, 1965-1977 (1973).

[28] M. G. T. Mentink, M. M. J. Dhalle, D. R. Dietderich, A. Godeke, W. Goldacker, F. Hellman, and H. H. J. ten Kate, "Towards analysis of the electron density of states of Nb3Sn as a function of strain", AIP Conf. Proc. 1435, 225-232 (2012).

[29] H. Devantay, J. L. Jorda, M. Decroux, and J. Muller, "The physical and structural properties of superconducting A15-type Nb-Sn alloys", J. of Mat. Sc. 16, 2145 - 2154 (1981).

[30] J. Zhou, Y. Jo, Z. H. Sung, H. Zhou, P. J. Lee, and D. C. Larbalestier, "Evidence that the upper critical field of $\mathrm{Nb}_{3} \mathrm{Sn}$ is independent of whether it is cubic or tetragonal", Appl. Phys. Lett. 99, 122507 (2011).

[31] W. Goldacker and R. Flükiger, "Phase transitions and superconducting properties of binary and Ti, Ta, Ga and H alloyed $\mathrm{Nb}_{3}$ Sn", Physica 135B, 359-363 (1985).

[32] R. Flükiger, C. Senatore, M. Cesaretti, F. Buta, D. Uglietti, and B. Seeber, "Optimization of $\mathrm{Nb}_{3} \mathrm{Sn}$ and $\mathrm{MgB}_{2}$ wires", Superc. Sci. Techn. 21, 054015 (2008).

[33] V. Guritanu, W. Goldacker, F. Bouquet, Y. Wang, R. Lortz, G. Goll, and A. Junod, "Specific heat of $\mathrm{Nb}_{3} \mathrm{Sn}$ : The case for a second energy gap", Phys. Rev. B 70, 184526 (2004).

[34] J. Labbé and J. Friedel, "Effect of temperature on the electronic instability and change in the crystal phase in low temperature $\mathrm{V}_{3} \mathrm{Si}$ ", J. de Phys. 27, 303 (1966).

[35] B. Sadigh and V. Ozolins, "Structural instability and electronic excitations in $\mathrm{Nb}_{3} \mathrm{Sn}$, Phys. Rev. B 57, 2793 - 2800 (1998).

[36] W. Weber and L. F. Mattheiss, "Electronic structure of tetragonal Nb 33 Sn", Phys. Rev. B 25, 2270 - 2284, (1982).

[37] G. Shirane and J. D. Axe, "Neutron Scattering Study of the Lattice-Dynamical Phase Transition in $\mathrm{Nb}_{3} \mathrm{Sn}$ ", Phys. Rev. B 4, 2957 - 2963 (1971).

[38] L. F. Mattheiss and L. R. Testardi, "Plasma energies for A-15 compounds", Phys. Rev. B 17, 4640 - 4643 (1978). 
[39] P. B. Allen, W. E. Pickett, K. M. Ho, and M. L. Cohen, "Anomalous Resistivities of A15 Metals - Insights from Band Theory", Phys. Rev. Lett. 40, 1532 - 1534 (1978).

[40] P. B. Allen, T. P. Beaulac, and F. S. Khan, "DC transport in metals", Phys. Rev. B 34, 4331 4333 (1986).

[41] P. B. Allen, W. E. Pickett, and H. Krakauker, "Anistropic normal-state transport properties predicted and analyzed for high- $T_{\mathrm{c}}$ oxide superconductors", Phys. Rev. B 37, 7482 - 7490 (1988).

[42] W. E. Pickett and P. B. Allen, "Optical properties of $\mathrm{Nb}_{3} \mathrm{Sn}$ : A probe of the electronic density of states", Sol. St. Comm. 12, 677-680 (1973).

[43] K. C. Lim, J. D. Thompson, and G. W. Webb, "Electronic density of states and $T_{\mathrm{c}}$ in $\mathrm{Nb}_{3} \mathrm{Sn}$ under pressure", Phys. Rev. B 27, 2781 - 2787 (1983).

[44] S. Kartha, J. A. Krumhansl, J. P. Sethna, and L. K. Wickham, "Disorder-driven pretransitional tweed pattern in martensitic transformations", Phys. Rev. B. 52, 803 - 821 (1995).

[45] M. J. Goringe and U. Valdre, "Transmission electron microscopy of Vanadium-Silicon $\left(\mathrm{V}_{3} \mathrm{Si}\right)$ at low temperatures", Proc. Roy. Soc. A 295, 192 - 216 (1966).

[46] T. Onozuka, N. Ohnishi, and M. Hirabayashi, "Low temperature electron microscopy on the cubictetragonal transformation of $\mathrm{V}_{3} \mathrm{Si}$ ", Met. Trans. A 19A, 797 - 801 (1988).

[47] J. D. Axe and G. Shirane, "Inelastic-neutron-scattering study of (111) LA phonons in $\mathrm{Nb}_{3} \mathrm{Sn}$, Phys. Rev. B 28, 4829 - 4830, (1973).

[48] L. Pintschovius, H. Takei and N. Toyota, "Phonon Anomalies in $\mathrm{Nb}_{3} \mathrm{Sn}$, Phys. Rev. Lett. 54, 1260 - 1263 (1985).

[49] F. Hellman and T. Geballe, "Specific heat of thin-film A15 superconductors: An anomalous inhomogeneity discovered", Phys. Rev. B 36, 107-120 (2005).

[50] M. G. T. Mentink, J. E. Bonevich, M. M. J. Dhalle, D. R. Dietderich, A. Godeke, F. Hellman, H. H. J. ten Kate, "Superconductivity in Nb-Sn thin films of stoichiometric and off-stoichiometric compositions", IEEE trans. on Appl. Supercond. 23, 7100505 (2013).

[51] A. Godeke, "A review of the properties of $\mathrm{Nb}_{3} \mathrm{Sn}$ and their variation with A15 composition, morphology and strain state", Superc. Sci. Techn. 19, R68-80 (2006).

[52] R. Flükiger, "Growth of A15 type single crystals and polycrystals and their physical properties", Handbook of supercond. mat. 1, IOP publishing ltd., Bristol (2003).

[53] D. F. Moore, R. B. Zubeck, and J. M. Rowell, "Energy gaps of the A-15 superconductors $\mathrm{Nb}_{3} \mathrm{Sn}$, $\mathrm{V}_{3} \mathrm{Si}$, and $\mathrm{Nb}_{3}$ Ge measured by tunneling", Phys. Rev. B 20, 2721 - 2738 (1979).

[54] D. A. Rudman, F. Hellman, R. H. Hammond, and M. R. Beasley, "A15 Nb-Sn tunnel junction fabrication and properties", J. Appl. Phys. 55, 3544 - 3553 (1984).

[55] V. L. Ginzburg, and L. D. Landau, Zh. Eksp. Teor. Fiz. 20, 1064 (1950).

[56] A. A. Abrikosov, Zh. Eksp. Teor. Fiz. 32, 1442 (1957).

[57] L. P. Gorkov, Zh. Eksp. Teor. Fiz. 36, 1918 (1959).

[58] P. G. De Gennes, "Superconductivity of Metals And Alloys", Perseus Books, New York City (1999).

[59] E. Helfand and N. R. Werthamer, "Temperature and Purity Dependence of the Superconducting Critical Field, $H_{\mathrm{c} 2}$ ", Phys. Rev. Lett. 13, 686 - 688 (1964).

[60] E. Helfand and N. R. Werthamer, "Temperature and Purity Dependence of the Superconducting Critical Field, $H_{\mathrm{c} 2}$. II", Phys. Rev. 147, 288 - 294 (1966).

[61] P.C. Hohenberg and N. R. Werthamer, "Anisotropy and Temperature Dependence of the Upper Critical Field of Type-II Superconductors", Phys. Rev. 153, 493 - 497 (1967).

[62] N. R. Werthamer, E. Helfand, and P. C. Hohenberg, "Temperature and Purity Dependence of the Superconducting Critical Field, $H_{\mathrm{c} 2}$. III. Electron Spin and Spin-Orbit Effects", Phys. Rev. 147, 295 - 302 (1966).

[63] N. Schopohl and K. Scharnberg, "Upper Critical Fields in the Presence of Electron-Spin and Spin-Orbit Effects", Physica 107 B, 293-294 (1981).

[64] C. T. Rieck, K. Scharnberg, and N Schopohl, "Quasiclassical Theory of the Upper Critical Field of High-Field Superconductors. Application to Momentum-Dependent Scattering", J. of Low Temp. 
Phys. 84, 381 - 464 (1991).

[65] N. Schopohl and K. Scharnberg, "Effect of Anisotropic Scattering on the Upper Critical Field of High-Field Superconductord", Physica 135B, 482-485 (1985).

[66] T. P. Orlando and M. R. Beasley, "Pauli limiting and the Possibility of Spin Fluctuations in the A15 Superconductors", Phys. Rev. Lett. 46, 1598 - 1601 (1981).

[67] N. R. Werthamer and W. L. McMillan, "Temperature and Purity Dependence of the Superconducting Critical Field $H_{\mathrm{c} 2}$. IV. Strong-Coupling Effects", Phys. Rev. 158, 415 - 417 (1967).

[68] N. F. Masharov, "Properties of Superconductors with Strong Coupling close to their Critical Temperature in Arbitrary Magnetic Fiedlds", Fiz. Tverd. Tela. 16, 2343-2349 (1974).

[69] M. Schossmann and E. Schachinger, "Strong-coupling theory of the upper critical magnetic field $H_{\mathrm{c} 2}$ ", Phys. Rev. B 33, 6123 - 6131 (1986).

[70] M. Schossmann and E. Schachinger, "Theory of $H_{\mathrm{c} 2}$ for superconductors with energy-dependent electronic density of states", Phys. Rev. B 30, 1349 - 1356 (1984).

[71] A. Godeke, M. C. Jewell, C. M. Fischer, A. A. Squitieri, P. J. Lee, and D. C. Larbalestier, "The upper critical field of filamentary $\mathrm{Nb}_{3} \mathrm{Sn}$ conductors", J. of Appl. Phys. 97, 093909 (2005).

[72] M. G. T. Mentink, M. M. J. Dhalle, D. R. Dietderich, A. Godeke, W. Goldacker, F. Hellman, H. H. J. ten Kate, M. D. Sumption, M. A. Susner, "The effect of Ta and Ti additions on the strain sensitivity of bulk Niobium-Tin", Phys. Proc. 36, 491-496 (2012).

[73] M. C. Jewell, A. Godeke, P. J. Lee and D. C. Larbalestier, "The Upper Critical Field of Stoichiometric and Off-Stoichiometric Bulk, Binary $\mathrm{Nb}_{3} \mathrm{Sn}^{\prime}$, Adv. in Cryo. Eng 711, 474 - 484 (2004).

[74] S. Foner and E. J. McNiff Jr., "Upper critical fields of cubic and tetragonal single crystal and polycrystalline $\mathrm{Nb}_{3} \mathrm{Sn}$ in DC fields to 30 Tesla", Sol. St. Comm. 39, 959-964 (1981).

[75] M. Naus, "Optimization of Internal-Sn $\mathrm{Nb}_{3} \mathrm{Sn}$ Composites", $\mathrm{PhD}$ Thesis, University of Wisconsin - Madison (2002).

[76] A. L. Fetter and P. C. Hohenberg, "Theory of Type II Superconductors", Superconductivity vol. 2, Marcel Dekker inc, New York (1969).

[77] D. C. Larbalestier, private communication (2015).

[78] K. H. Bennemann and J. W. Garland, "Theory for Superconductivity in d-Band Metals", AIP Conf. Proc. 4, pp. 103 - 137 (1972). 